\title{
The GMRT High Resolution Southern Sky Survey for pulsars and transients -I. Survey description and initial discoveries
}

\author{
B. Bhattacharyya ${ }^{1}$, S. Cooper ${ }^{1}$, M. Malenta ${ }^{1}$, J. Roy ${ }^{1,2}$, J. Chengalur ${ }^{2}$, M. Keith ${ }^{1}$, \\ S. Kudale ${ }^{2}$, M. McLaughlin ${ }^{3}$, S. M. Ransom ${ }^{4}$, P. S. Ray ${ }^{5}$, B. W. Stappers ${ }^{1}$
}

\begin{abstract}
We are conducting a survey for pulsars and transients using the Giant Metrewave Radio Telescope (GMRT). The GMRT High Resolution Southern Sky (GHRSS) survey is an off-Galactic-plane $(|b|>5)$ survey in the declination range $-40^{\circ}$ to $-54^{\circ}$ at $322 \mathrm{MHz}$. With the high time (up to $30.72 \mu \mathrm{s}$ ) and frequency (up to $0.016275 \mathrm{MHz}$ ) resolution observing modes, the $5 \sigma$ detection limit is 0.5 mJy for a $2 \mathrm{~ms}$ pulsar with $10 \%$ duty cycle at $322 \mathrm{MHz}$. Total GHRSS sky coverage of $2866 \mathrm{deg}^{2}$, will result from 1953 pointings, each covering $1.8 \mathrm{deg}^{2}$. The $10 \sigma$ detection limit for a $5 \mathrm{~ms}$ transient burst is $1.6 \mathrm{Jy}$ for the GHRSS survey. In addition, the GHRSS survey can reveal transient events like rotating radio transients or fast radio bursts. With $35 \%$ of the survey completed (i.e. 1000 $\operatorname{deg}^{2}$ ), we report the discovery of 10 pulsars, one of which is a millisecond pulsar (MSP), one of the highest pulsar per square degree discovery rates for any off-Galactic plane survey. We re-detected 23 known in-beam pulsars. Utilising the imaging capability of the GMRT we also localised 4 of the GHRSS pulsars (including the MSP) in the gated image plane within $\pm 10^{\prime \prime}$. We demonstrated rapid convergence in pulsar timing with a more precise position than is possible with single dish discoveries. We also exhibited that we can localise the brightest transient sources with simultaneously obtained lower time resolution imaging data, demonstrating a technique that may have application in the SKA.
\end{abstract}

\footnotetext{
${ }^{1}$ Jodrell Bank Centre for Astrophysics, School of Physics and Astronomy, The University of Manchester, Manchester M13 9PL, UK

${ }^{2}$ National Centre for Radio Astrophysics, Tata Institute of Fundamental Research, Pune 411 007, India

${ }^{3}$ Department of Physics \& Astronomy, West Virginia University, Morgantown, WV 26506, US

${ }^{4}$ National Radio Astronomy Observatory(NRAO), Charlottesville, VA 22903, USA

${ }^{5}$ Space Science Division, Naval Research Laboratory, Washington, DC 20375-5352, USA
} 


\section{Introduction}

Pulsars are rapidly rotating neutron stars emitting a beam of radio waves from their magnetic poles, that sweep our line-of-sight at the spin period. In the 48 years since the discovery of pulsars, the present population of about 2300 known pulsars have spin periods ranging from $1.4 \mathrm{~ms}$ to $8.5 \mathrm{~s}$, magnetic field strengths from $6.6 \times 10^{7} \mathrm{G}$ to $1.2 \times 10^{13} \mathrm{G}$, line-of-sight electron column density values, called dispersion measures (DMs), from 2.38 $\mathrm{pc} \mathrm{cm}^{-3}$ to $1456 \mathrm{pc} \mathrm{cm}^{-3}$, flux densities at $400 \mathrm{MHz}$ ranging from $0.1 \mathrm{mJy}$ to $5000 \mathrm{mJy}$ and characteristic ages ranging from $2.2 \times 10^{2}$ years to $6.7 \times 10^{10}$ years ${ }^{6}$. In an attempt to understand the distribution of sources within the limits of the known population and to probe the limits themselves there are many ongoing surveys that are discovering pulsars at an encouraging rate. Although many of the newly discovered pulsars have similar properties to the known population, some of the new ones are pushing the boundary of known parameter space. For example Low-mass X-ray binary-radio millisecond pulsar transitioning system: J1023+0038 (Archibald et al. 2009) and J1227-4853 (Roy et al. 2015), a hierarchical stellar triple pulsar system: J0337+1715 (Ransom et al. 2014), a massive neutron star-white dwarf binary: J0348+0432 (Antoniadis et al. 2013), and a pulsar with a very dense planetary mass companion: J1719-1438 (Bailes et al. 2011). The diversity of properties of these objects justifies the requirement of having more sensitive surveys for further discoveries.

Modeling by Faucher-Giguere et al. (2006) indicates that the Galaxy contains about $10^{5}$ pulsars and the number of presently known pulsars is only around $1 \%$ of this population. This implies that a vast majority of pulsars are waiting to be discovered. Studies of pulsars yield a better understanding of a variety of physics problems, from acceleration of particles in ultra strong magnetic fields (primarily via study of emission properties of normal pulsars, having spin period $>30 \mathrm{~ms}$ ) to probes of ultra dense matter (mostly via studying the timing properties of millisecond pulsars, having spin period $<30 \mathrm{~ms}$, that are very stable rotators). Pulsars provide useful probes of their environments, e.g. inside pulsar wind nebulae, the centre of Globular clusters, or the Galactic centre. Some of the normal pulsars are useful for investigation of single pulse behaviour and can exhibit interesting individual properties, like glitches (Lyne et al. 1996), profile state changes (Lyne et al. 2010), nulling (Backer 1970) and intermittency (Kramer et al. 2006). The emission mechanism for pulsar radiation at radio wavelengths is not yet understood, and a range of theoretical models have been proposed (e.g. Ruderman et al. (1975); Gil et al. (2003); Alexander et al. (2014)) to explain it. The discovery of a new sample of pulsars with diverse observational properties will provide additional constraints on the possible emission mechanism. Additional pulsars distributed

\footnotetext{
${ }^{6}$ http://www.atnf.csiro.au/people/pulsar/psrcat/
} 
in our Galaxy will aid the investigation of properties of the interstellar medium via scattering measurements as well as via dispersion measure and rotation measure studies. The fractional rotational stability of millisecond pulsars (MSPs), one part in $10^{15}$, is comparable to atomic clocks (Lorimer et al. 2004). Such rotational stability, compactness second only to black holes, and their presence in binary systems, make MSPs ideal laboratories to test the physics of gravity and as detectors for long-wavelength gravitational waves (Detweiler et al. 1979). Some relativistic binary MSPs are useful for tests of gravity and an array of spatially distributed MSPs can be used to detect gravitational waves (Lee et al. 2012). In addition to the contribution towards the detection of gravitational wave signal, MSP evolutionary processes can be tracked through individual interesting discoveries of MSPs in special evolutionary phases. For example, the black-widow systems (Roberts et al. 2011) provides a missing link between the binary and isolated MSPs and Low-mass X-ray binary-radio MSP transitioning redback systems (Archibald et al. 2009; Roy et al. 2015) provides a probe of binary evolution.

In addition to the regular emission from pulsars, a wide variety of transient phenomena are known at faster time scales (micro-seconds to seconds). The discoveries of giant pulses from pulsars (Lundgren et al. 1995), quasi-periodic emission from rotating radio transients (RRATs; McLaughlin et al. (2006)), bursts of periodic pulsations from magnetars (Camilo et al. 2006) and highly dispersed fast radio bursts (FRBs) of possible extragalactic origin (Lorimer et al. 2007) are a few examples. The FRBs have been discovered in radio pulsar surveys at Parkes and Arecibo in the last decade (Lorimer et al. 2007; Thornton et al. 2013; Spitler et al. 2014; Burke-Spolaor \& Bannister et al. 2014; Petroff et al. 2014). All FRBs discovered to date have been single radio events of millisecond duration with DM values generally higher than the possible Galactic contribution. Majority of the FRB population theories suggest that they originate at cosmological distances (Deng et al. (2014); Luan et al. (2014); Keane \& Petroff (2015)), although the actual progenitors are still unknown. The probable extragalactic origin allows these bursts to be used for determination of the baryon content of the intergalactic medium. The detection of a large number of FRBs (presently only $17 \mathrm{FRB} \mathrm{f}^{7}$ are known) is required in order for us to have a better understanding of the nature of the population. This will allow one to establish whether they form a population of standard candles or some other distribution. Also many lines-of-sights will be needed to make them useful probes of the intergalactic medium and for determining the presence of the so-called missing baryons. With a larger population of sources it will also be possible to determine the spectral index of the radio emission which is a key element for probing the emission physics. Magnetar flares (Thornton et al. (2013); Kulkarni et al. (2014)), blitzars

\footnotetext{
${ }^{7}$ https://astro.uni-bonn.de/ tauris/NS2015/Keane_FRBs.pdf
} 
(Falcke et al. 2014), super-giant pulses from neutron stars (Cordes et al. 2015), and pulsarplanet systems (Mottez \& Zarka 2014), are some of the models suggesting extragalactic origin of FRBs at cosmological distances.

The known pulsar population is increasing with the surveys running at major single dish and array telescopes around the world: e.g. High Time Resolution Universe (HTRU) survey at Parkes (Keith et al. 2010), HTRU-North survey at Effelsberg (Barr et al. 2011), SUrvey for Pulsars and Extragalactic Radio Bursts (SUPERB) survey ${ }^{8}$ at Parkes, Pulsar survey Arecibo L-band Feed Array (PALFA, Cordes et al. (2006)), Green Bank Telescope drift scan survey (Boyles et al. 2013), Green Bank Northern Celestial Cap (GBNCC) survey (Stovall et al. 2014), Arecibo all-sky 327 MHz drift pulsar survey (AO327, Deneva et al. (2013)), the LOFAR Pulsar Pilot Survey (LPPS) survey (Coenen et al. 2014), the LOFAR Tied-Array All-Sky Survey (LOTAAS) ${ }^{9}$ at LOFAR and Fermi directed MSP surveys (Ray et al. 2012).

Because of the generally steep spectral nature of pulsars, lower frequencies are an obvious choice for searching for fainter pulsars away from the Galactic plane, where search sensitivity is not severely affected by sky temparature and increased scattering. Such surveys away from the Galactic plane will detect relatively older pulsars. The success of Fermi directed searches in discovering more than $60 \%$ of Fermi MSP 10 at frequencies below $1 \mathrm{GHz}$ demonstrates the advantages of low frequency searches (Ray et al. 2012). Besides the GBT and the LOFAR, the Giant Metrewave Radio Telescope (GMRT ${ }^{11}$ ) is another facility which has a sensitive low frequency $(<600 \mathrm{MHz}$ ) observing capability. With the aid of reduced quantised noise supported by the flexible GMRT Software Backend, (GSB; Roy et al. (2010)) the search sensitivity to pulsars in general is improved by $30 \%$ compared to its old hardware counterpart. An additional 30\% increase in sensitivity to MSPs is achieved through using the higher time resolution mode implemented in the GSB. Thus, effectively, the search sensitivity to normal pulsars is improved by $30 \%$ whereas for MSPs a $60 \%$ improved sensitivity is achieved with the GSB (Roy et al. 2013). This is exemplified by the discovery of seven MSPs from Fermi directed searches by Bhattacharyya et al. (2013). These were the first Galactic MSPs discovered with the GMRT. This clearly illustrates the potential of low frequency pulsar search using the GMRT.

\footnotetext{
${ }^{8}$ https://sites.google.com/site/publicsuperb/

${ }^{9} \mathrm{http}: / /$ www.astron.nl/lotaas

${ }^{10} \mathrm{http}$ ///astro.phys.wvu.edu/GalacticMSPs/GalacticMSPs.txt

${ }^{11}$ http://gmrt.ncra.tifr.res.in
} 
Since we have no information on most pulsar positions a priori (unlike the ones associated with unidentified Fermi sources or supernovae remnants), blind searches are required for pulsar discoveries. Lower frequency surveys benefit from a larger field-of-view, in addition to the steep spectral nature of pulsars. The success of targeted searches with the GMRT makes it evident that blind searches with the GMRT are warranted and can produce a significant science yield in terms of discovering new pulsars, MSPs and transient events. Thus we are carrying out the GMRT High Resolution Southern Sky (GHRSS ${ }^{12}$ ) survey for pulsars and transients to conduct one of the most sensitive and highest resolution surveys for the southern hemisphere. To make the search more efficient and complementary we target a part of the southern sky which has not been searched at frequencies below $1.4 \mathrm{GHz}$ for the last two decades. New observing modes were developed to increase the time and frequency resolution of the survey, making it even more sensitive to MSPs and normal pulsars with higher DM values.

Large uncertainties associated with the discovery positions (e.g. $\pm 40^{\prime}$ for GMRT-322, $\pm 18^{\prime}$ for GBT $-350, \pm 7^{\prime}$ for Parkes-Lband) hinder sensitive follow up studies of these newly discovered pulsars using coherent beams of array telescopes, or at higher frequencies using single dishes. The GMRT interferometric array allows us to localise the newly discovered pulsars and transients in the image plane with an accuracy of better than $\pm 10^{\prime \prime}$ (half of the typical synthesized beam used in the image made at $322 \mathrm{MHz}$ ). Precise a priori astrometric positions are also needed to overcome the effect of large covariances in the timing fits (with discovery position, pulsar period derivative and unknown binary model in case of binary pulsars) for rapid convergence of an initial timing model. Moreover, sensitive coherent array follow up observations can significantly reduce the use of telescope time by $\sim 20 \times$ for the GMRT (due to the 4 to 5 times sensitivity improvement for coherent array observations) and also improves the uncertainties in time-of-arrivals (TOAs) or generates more closely spaced TOAs in order to avoid ambiguous phase connection while timing.

Finding pulsars is only half the job. The other half is to understand their nature, rotation properties, spectral indices and relation to the pulsar population; for this regular follow up is needed. Precise measurements of these parameters puts a new discovery in its proper place in the pulsar population. Equipped with the precise localisation we carry out more sensitive follow up observations with the coherent array of the GMRT. In this paper we demonstrate that convergence in the timing fit is facilitated by a priori knowledge of a precise position.

All the known FRBs detected to date have been found with single dish telescopes, and

\footnotetext{
${ }^{12}$ http://www.jb.man.ac.uk/research/pulsar/Resources/ghrss.html
} 
for most of them the uncertainty in position is of order of $\pm 7^{\prime}$ or more. Interferometric imaging using the simultaneously recorded visibility data at $2 \mathrm{~s}$ interval will allow precise localisation of the FRBs detected from the GHRSS survey. This will allow multi-wavelength follow up and thus the possibility of identification of putative host galaxies. However, this will only be feasible for the relatively stronger ( $>3 \mathrm{Jy}$ ) (detailed in Section 9.2) FRBs. Thus we will be able to localise bright FRBs (e.g. Lorimer bursts, Lorimer et al. (2007)).

In this paper we describe the survey, the data analysis pipeline, discovery parameters of the 10 pulsars, the localisation of 4 and timing solution of 2 pulsars discovered in the GHRSS survey. The observing system and target sky are detailed in Section 2 and Section 3 of the paper. The prediction of survey outcome is detailed in Section 4. The survey processing is discussed in Section 5. In Section 6 we calculate the theoretical sensitivity and compare that with the sensitivity achieved in the survey. Section 7 describes the re-detection of known pulsars from this survey and their interferometric flux measurements. In Section 8 we give the basic parameters of the pulsars discovered in the GHRSS survey and in Section 9 we detail the localisation of detected events using the GMRT interferometric array. The timing and follow up of the pulsars discovered in the GHRSS survey is described in Section 10. The relevance of GHRSS survey results and methods with the future Square Kilometre Array (SKA) is discussed in Section 11. Section 12 presents the summary of this paper.

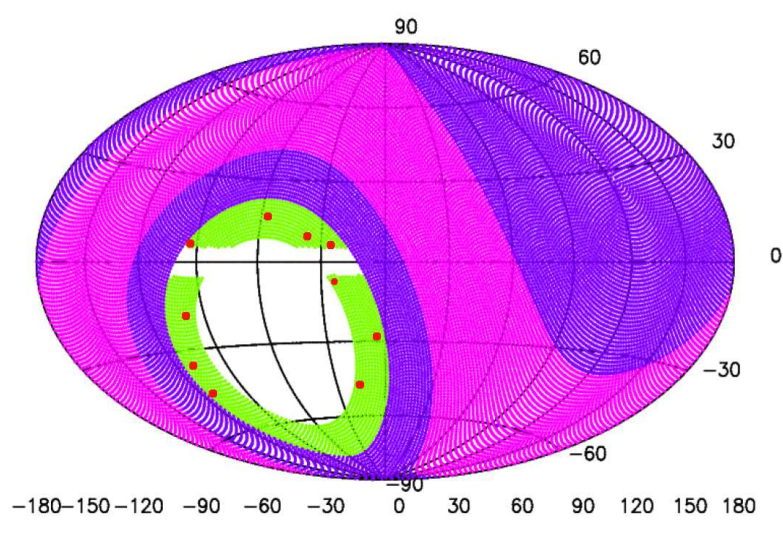

(a)

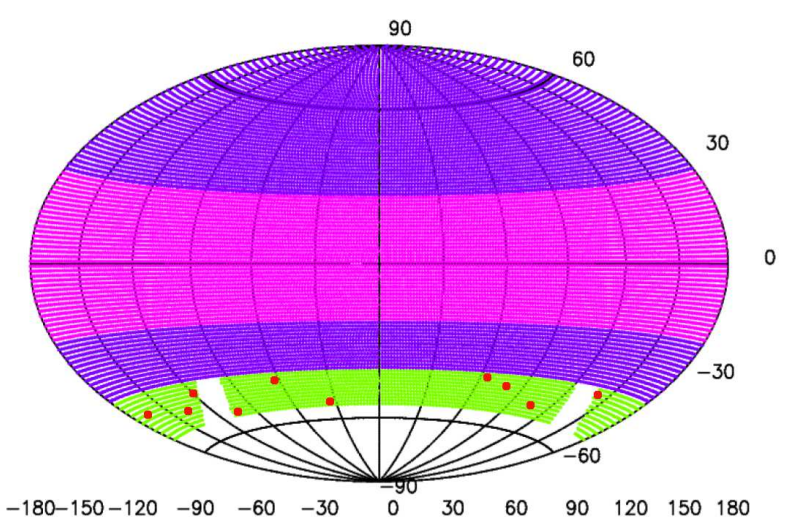

(b)

Fig. 1.- (a) Sky coverage in Galactic coordinates of recent and ongoing pulsar surveys around $300 \mathrm{MHz}$; GBT drift scan (pink) at $350 \mathrm{MHz}$, GBNCC (purple) at $350 \mathrm{MHz}$ and GHRSS (green) at $322 \mathrm{MHz}$. Red dots are the pulsars discovered with the GHRSS survey. (b) Sky coverage in RA and DEC. 


\section{Observing system}

The GMRT is a multi-element aperture synthesis telescope consisting of 30 antennas, each of $45 \mathrm{~m}$ diameter, spread over a region of $25 \mathrm{~km}$ diameter and operating at 5 different wave bands from $150 \mathrm{MHz}$ to $1450 \mathrm{MHz}$ (Swarup et al. 1997). The GMRT Software Backend, built using COTS components, is a fully real-time backend that supports a FX correlator and a beamformer for an array of 32 dual polarized signals, Nyquist sampled at 33 or $66 \mathrm{MHz}$ (Roy et al. 2010). We developed high time and frequency resolution observing modes that are ported to the GMRT Software Backend, producing incoherent or coherent beam $2048 \times 0.016275 \mathrm{MHz}$ filter-bank outputs sampled every $61.44 \mu \mathrm{s}$ (hereafter HiRes1) or $1024 \times 0.03255 \mathrm{MHz}$ sampled every $30.72 \mu \mathrm{s}$ (hereafter HiRes2). This translates to a data rate of $32 \mathrm{MB} / \mathrm{s}$ for the high time-resolution data with $8 \mathrm{bits} /$ sample after adding two polarized intensities. In parallel we recorded frequency averaged 512 channel visibilities for each of the 465 baselines at $2 \mathrm{~s}$ intervals resulting in a $2 \mathrm{MB} / \mathrm{s}$ data rate. The target scans were interleaved with scans on nearby calibrator sources every 1 hour to allow for phase calibration for imaging. The visibility data were then converted to FITs files for further processing in AIPS ${ }^{13} / \mathrm{CASA}^{14}$. The GMRT interferometric array provides the opportunity for combining the imaging mode with beamforming to widen the scope of studying pulsars (e.g. Roy et al. (2012); Bhattacharyya et al. (2013); Roy et al. (2013)). For the GHRSS survey we used the incoherent array beamformer output in order to maximise the field-of-view. In order to optimise the gain of the incoherent beam, the antenna based gain offsets were calibrated out prior to the addition of the signals.

Combining contributions from receiver temperature, ground temperature and spillover from the ground, the total system temperature at $322 \mathrm{MHz}$ is $106 \mathrm{~K}$ on cold sky ${ }^{15}$. The data were recorded to local disks and then transferred to the dedicated 64 TB GHRSS storage at the National Centre for Radio Astrophysics (NCRA) using an 8 Gbps fibrelink. We shipped the data disks from the GHRSS storage to the University of Manchester for processing on the Hydrus supercomputer. The Hydrus supercomputer with a dedicated 64 TB storage was used as a major processing and data-centre host for the GHRSS survey. Raw data were archived on LTO5 magnetic tapes immediately after the observations for long term storage.

\footnotetext{
${ }^{13}$ http://www.aips.nrao.edu/index.shtml

${ }^{14}$ http://casa.nrao.edu

${ }^{15}$ http://www.gmrt.ncra.tifr.res.in/gmrt_hpage/Users/doc/GMRT-specs.pdf
} 


\section{Sky coverage}

The $322 \mathrm{MHz}$ GHRSS survey consists of scans of the sky (ranging from $-40^{\circ}$ to $-54^{\circ}$ in declination), complementary to other low frequency surveys at the GBT, and LOFAR. Figure 1 shows portion of the target sky (marked in green) compared to the GBT surveys at 350 $\mathrm{MHz}$, noting that LOFAR is not scanning below declination zero. Though the pink and purple bands are indicative of GBT drift scan survey and GBNCC surveys, there are empty patches in the drift scan survey, which are being covered by the GBNCC survey.

The target sky for the GHRSS survey has not been searched at frequencies below $1.4 \mathrm{GHz}$ for the last two decades. The Parkes southern sky survey at $436 \mathrm{MHz}$ with $\sim 3 \mathrm{mJy}$ search sensitivity (Bailes et al. 1994) was conducted from 1991 to about 1995, and it discovered 85 normal pulsars and 17 MSPs 16 .

For the GHRSS survey we are not considering low Galactic latitudes $\left(|b|<5^{\circ}\right)$. The target sky has two components, a mid-latitude component, MGL, for $5<|b|<20^{\circ}$ covering the sky with declination range $-40^{\circ}$ to $-54^{\circ}$ with $1200 \mathrm{~s}$ integration with HiRes1 mode, and a high latitude component, HGL, $\left(|b|>20^{\circ}\right)$ covering the same declination range with $900 \mathrm{~s}$ integration with HiRes2 mode. The range of sky temperature for the GHRSS survey range from $33 \mathrm{~K}$ to $220 \mathrm{~K}$ based on the Haslam et al. (1982) $408 \mathrm{MHz}$ map.

In order to partly compensate for the $\sim 50 \%$ increase of sky temperature at MGL $(|b| \sim$ $10^{\circ}$ ) with respect to HGL, we choose to increase the integration time to $1200 \mathrm{~s}$. The target sky is covered with 682 and 911 pointings respectively for the MGL and HGL components, totaling 1953 pointing scans, each covering $1.8 \mathrm{deg}^{2}$ adding up to a total coverage of 2866 $\operatorname{deg}^{2}$. The survey parameters are summarised in Table 1.

\section{Survey prediction}

In order to determine the discovery potential of the GHRSS survey we performed simulations based on the PSRPOPPY ${ }^{17}$ code (Bates et al. 2014). PSRPOPPY is a modified version of PSRPOP (Lorimer et al. 2006) that models the period, luminosity and spatial distributions of a set of pulsars discovered in the successful Parkes Multibeam Pulsar Survey (Manchester et al. 2001) and the high-latitude pulsar survey (Burgay et al. 2006) to predict the discovery potential of the ongoing surveys (e.g. Keith et al. (2010); Keane et al. (2015)). For the

\footnotetext{
${ }^{16}$ http://www.jb.man.ac.uk/pulsar/research/jodsum/node3.html\#s70

${ }^{17}$ https://github.com/samb8s/PsrPopPy
} 

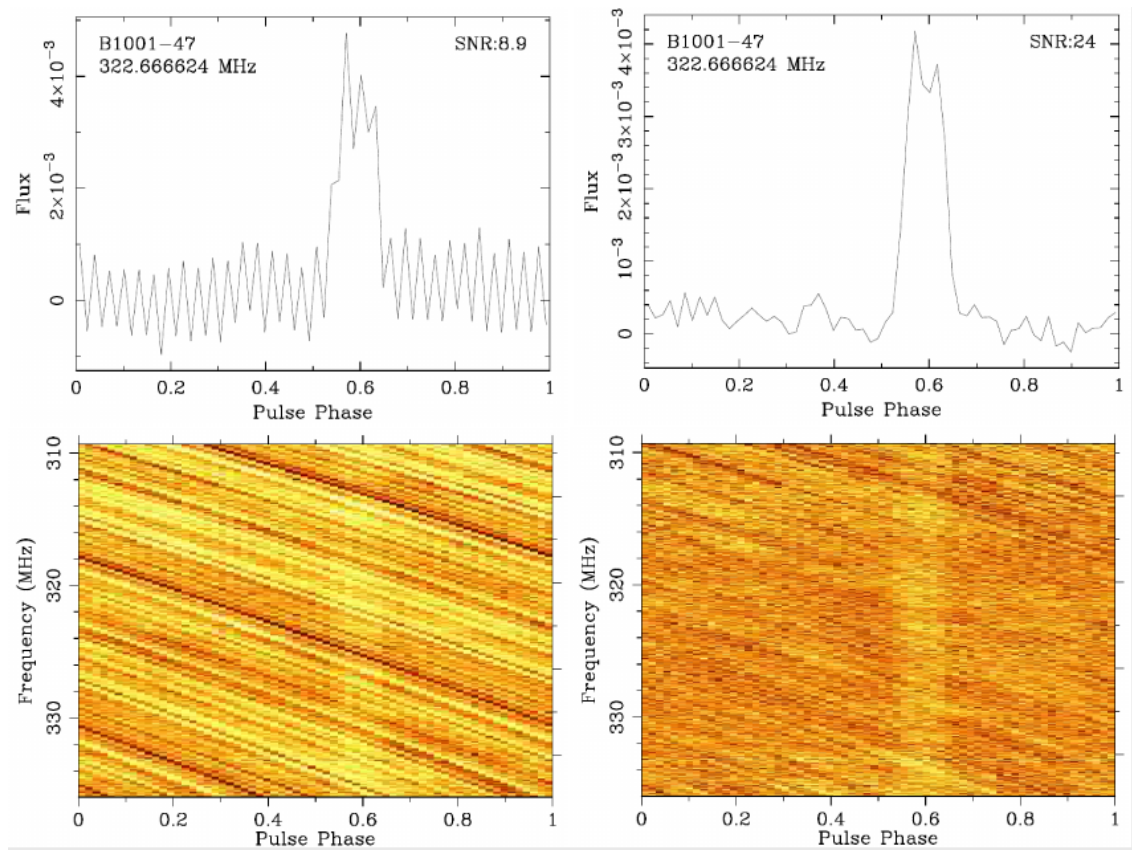

Fig. 2.- Integrated profile and phasogram of PSR B1007-47 for before (left panel) and after (right panel) zero-DM RFI mitigation. This observation was unusually affected by RFI. Even with some residual RFI present, a significant cleaning of the data and improvement of SNR by about a factor of 3 is achieved. The zero-DM RFI mitigation is followed by further cleaning of the data using the RFIFIND package of PRESTO resulting in 8\%-10\% improvement.

purpose of simulation we considered normal pulsars defined as $P>30 \mathrm{~ms}, \dot{P}<1 \times 10^{-12}$. In simulation of the expected normal pulsar population from the GHRSS survey, we used the input parameters listed in Table 3 (which are generally default options of PSRPOPPY and are similar to the ones used in Keith et al. (2010)).

Using the parameters listed in Table 3 and considering a total of 30,000 possibly detectable pulsars, the PSPRPOPPY software generates a model (Bates et al.2014) such that with Parkes Multibeam Pulsar Survey, 1038 pulsars are detected, which is close to the 1122 pulsars actually discovered in this survey. With this model, we ran the simulation 100 times with the specifications of the GHRSS survey and predict that it would discover $97 \pm 7$ pulsars, considering spectral index mean of -1.7 . Simulations considering different spectral indices with mean of -1.4 and -1.8 result in discovery predictions of about $81 \pm 6$ and $113 \pm 8$ normal pulsars respectively. We expect to find considerably fewer MSPs due to their intrinsic faint nature in addition to dispersion smearing and scatter broadening. For the simulation of the MSP population we considered a modified input for the spin period distribution, height above the Galactic plane and luminosity distribution as listed in Table 3. According to the 


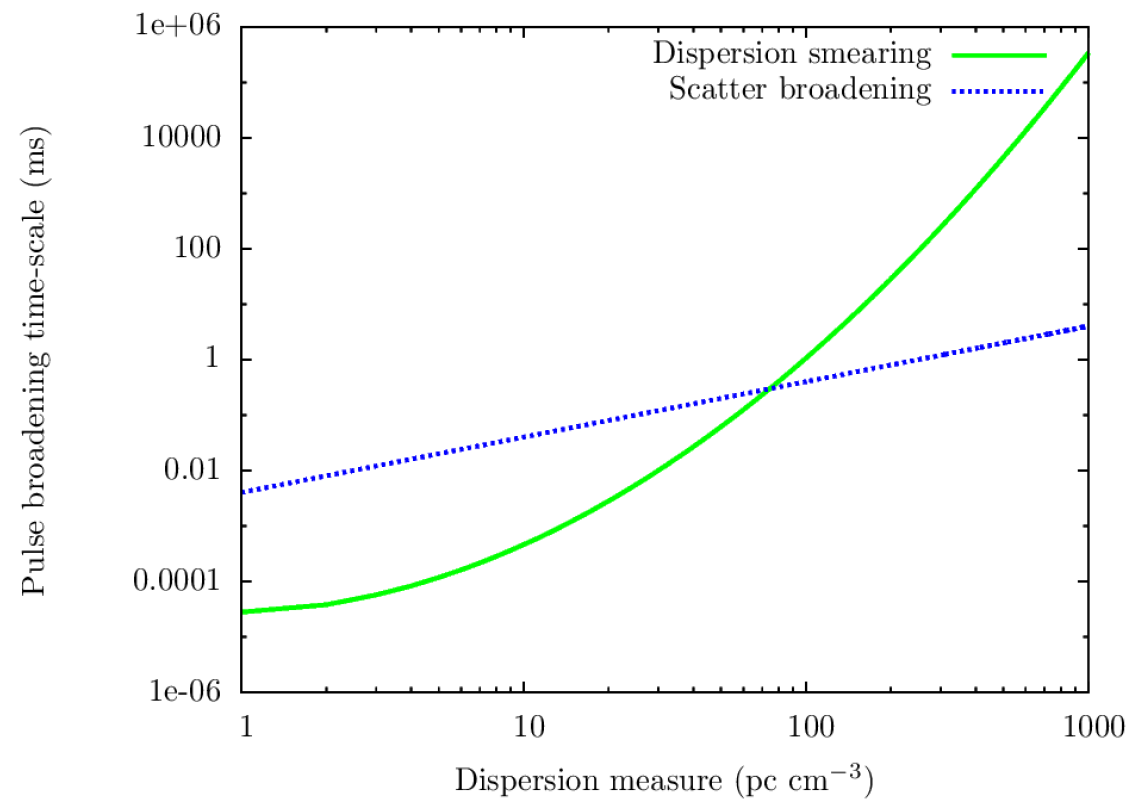

Fig. 3.- Scatter broadening and dispersion smearing as a function of DM. At DM $<75$ pc $\mathrm{cm}^{-3}$ scatter broadening dominates over dispersion smearing. Thus the GHRSS survey sensitivity is not significantly affected by the instrumental broadening up to DM of 75 pc $\mathrm{cm}^{-3}$.

PSRPOPPY simulation the GHRSS survey will discover around 9 \pm 3 MSPs considering spectral index mean of -1.7 . Simulations considering spectral indices with mean of -1.4 and -1.8 resulted in discovery predictions of about $7 \pm 3$ and $11 \pm 4$ MSPs respectively.

We also tried to judge the discovery potential of the GHRSS survey by comparing with the ongoing GBNCC survey which is scanning the sky at a similar frequency and with comparable sensitivity to the GHRSS survey i.e. about $0.5 \mathrm{mJy}$ of minimum detectable flux density (detailed in Section 6). GBNCC survey has covered $19500 \mathrm{deg}^{2}$ and has discovered 108 pulsar: ${ }^{18}$. Scaling from the GBNCC areal discovery rate, we expect to find of the order of 16 pulsars in the total GHRSS sky coverage $\left(2866 \mathrm{deg}^{2}\right)$.

In addition to the potential of discovering pulsars and millisecond pulsars, the GHRSS survey provides a snapshot of the transient radio sky in the southern hemisphere at 322 MHz. Based on the 35\% completion of HTRU-South survey analysis, Thornton et al. (2013) estimated the FRB rate as $1_{-0.5}^{+0.6} \times 10^{4} \mathrm{sky}^{-1}$ day $^{-1}$ for bursts having fluence $>3 \mathrm{Jy}$ ms. For

\footnotetext{
${ }^{18}$ http://arcc.phys.utb.edu/gbncc/
} 

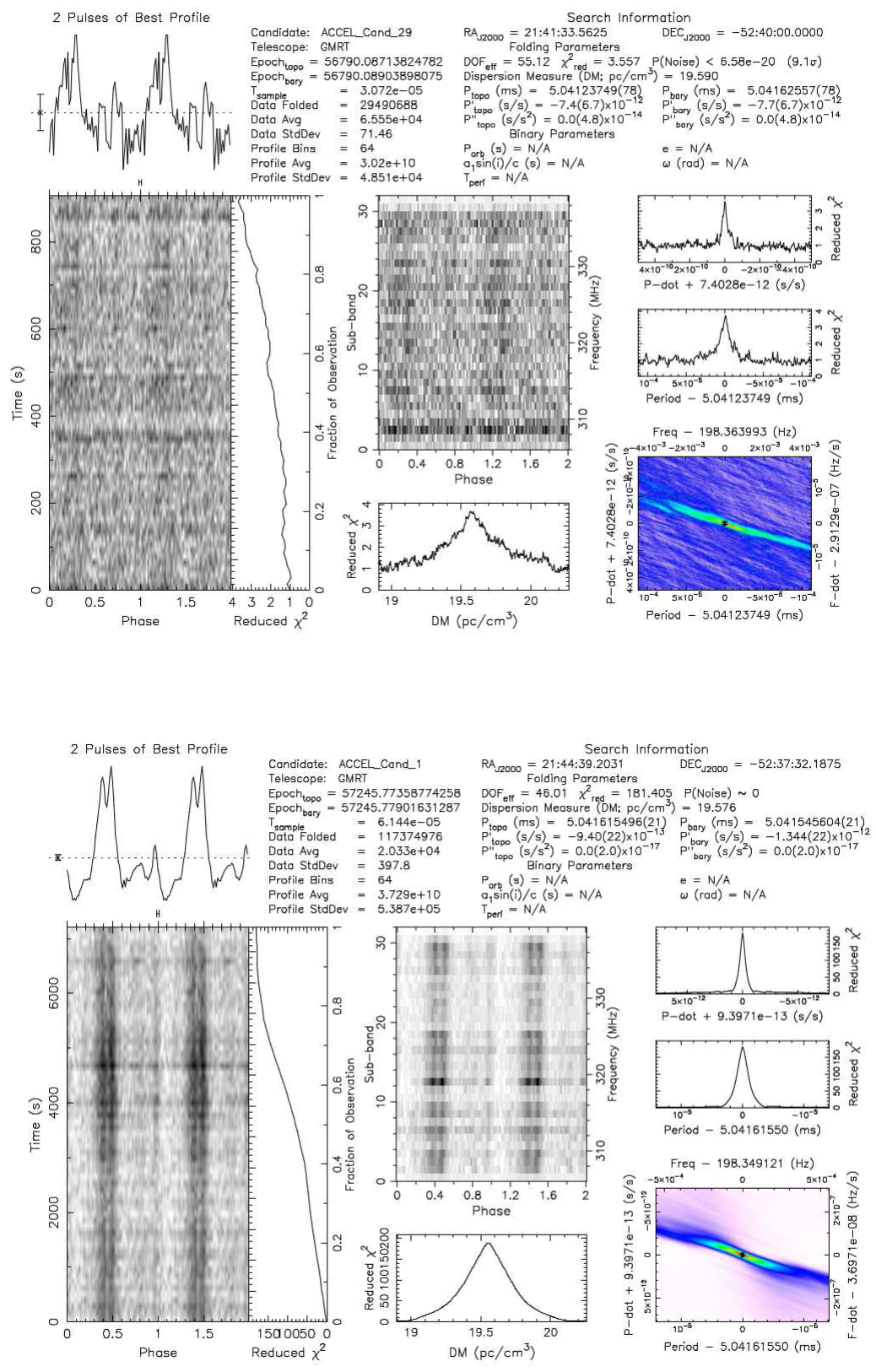

Fig. 4.- Top panel : Example diagnostic plot from the GHRSS periodicity search pipeline. This plot shows the MSP J2144-5237 discovered in the GHRSS survey with incoherent array observations, Bottom panel: PRESTO search output of PSR J2144-5237 with a coherent array observation. The plot demonstrates the improved detection significance with coherent array observations which can be performed after precise localisation of the pulsar in the image plane (Section 9p. 

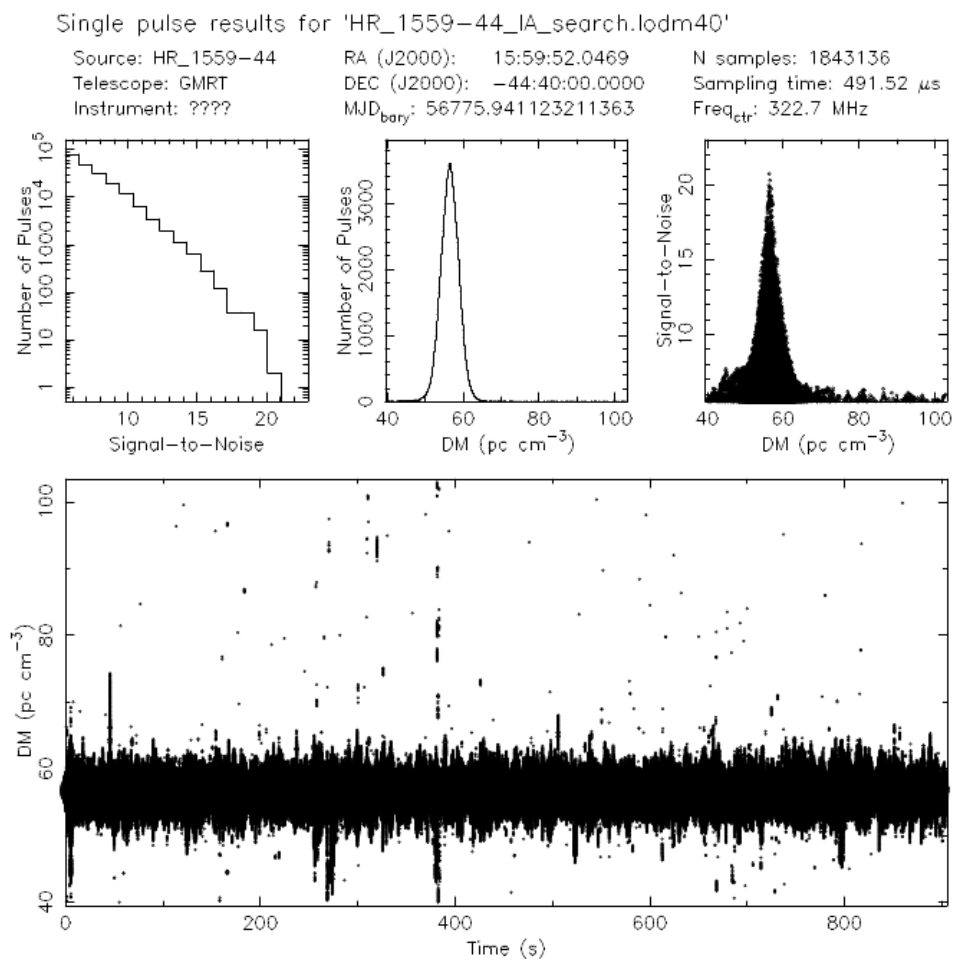

Fig. 5.- Example output of our PRESTO based single-pulse search pipeline using CPU processing, showing bright single pulses from PSR J1559-4438 (which is a known pulsar re-detected in the GHRSS survey) around a DM of $56 \mathrm{pc} \mathrm{cm}^{-3}$. The top left panel shows the signal-to-noise distribution of the detected pulses, number of pulses versus trial DM (in top centre), and signal-to-noise as a function of trial DM. The lower plot shows signal-to-noise of events as a function of time and trial DM.

the GHRSS survey we calculated a minimum detectable flux of 1.6 Jy for $10 \sigma$ detection with $5 \mathrm{~ms}$ bursts (detailed in Section 6). Coincidentally this minimum detectable flux for an FRB translates to a fluence of $\sim 3 \mathrm{Jy} \mathrm{ms}$. Considering the total $2866 \mathrm{deg}^{2}$ of the GHRSS survey area with $\sim 15$ mins of dwell time, we expect to discover $7_{-3}^{+5} \mathrm{FRBs}$ having fluence $>3 \mathrm{Jy}$ ms. However this prediction is based on the assumption that FRBs have a flat spectrum all the way from $1.4 \mathrm{GHz}$ to $300 \mathrm{MHz}$ and of course scattering and spectral index are largely unknowns for FRBs. Thus, the predictions are likely be lower than these estimates unless they are steep spectrum objects, and scattering at low frequencies is not large. Moreover the uncertainty on the rate presented by Thornton et al. (2013) and new lower rate estimates from Rane et al. (2015), Champion et al. (2015) and Petroff et al. (2014), the number of detected FRBs could be a factor of a few lower. However, any GHRSS detections will help 

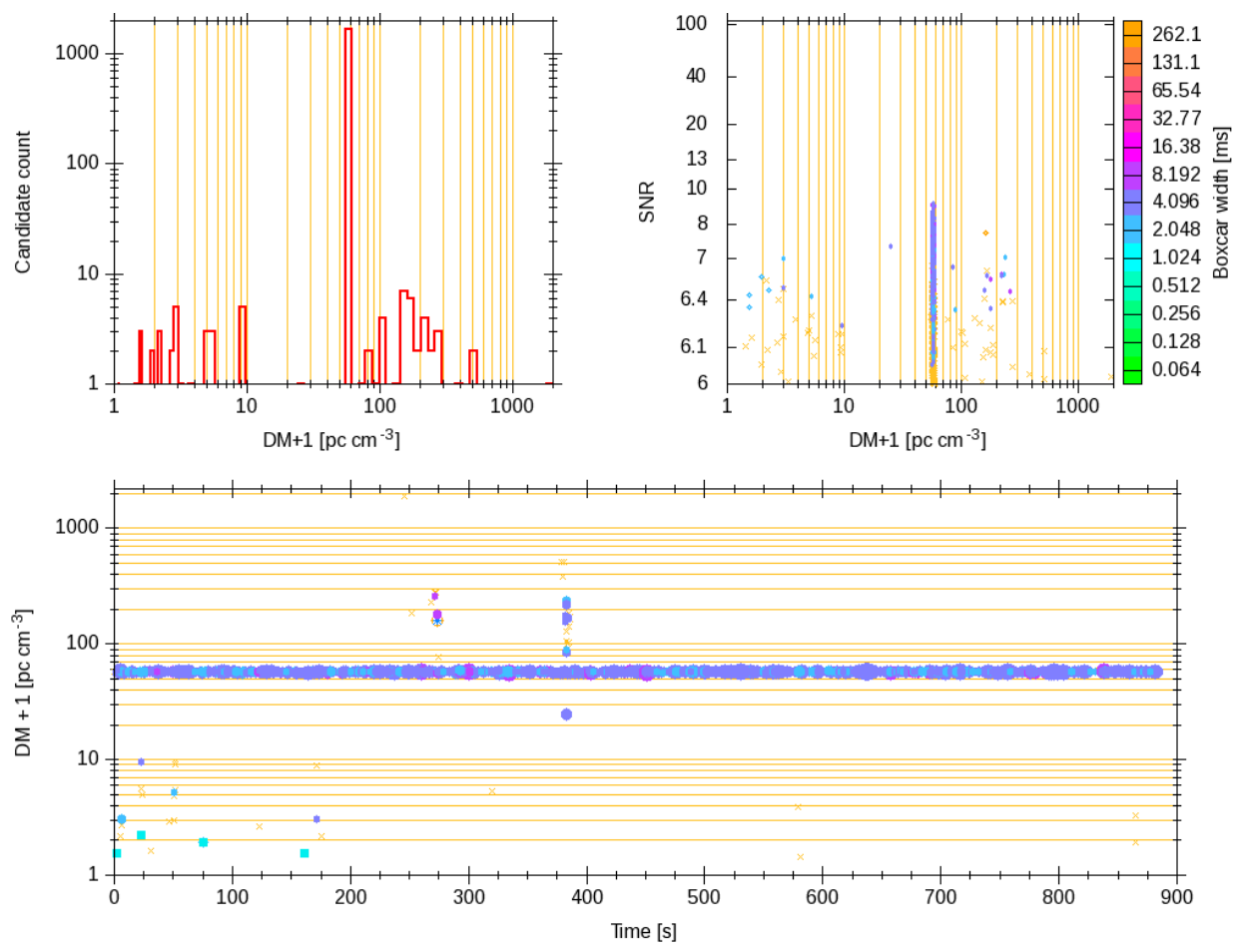

Fig. 6. - Example output of BIFORST single pulse search pipeline with GPU on the known pulsar J1559-4438 (re-detected in the GHRSS survey) showing bright pulses around a DM of $56 \mathrm{pc} \mathrm{cm}^{-3}$. The top left panel shows the detected pulses versus DM and top right panel shows signal-to-noise as a function of trial DM. The lower plot shows signal-to-noise of events as a function of time and trial DM.

us to significantly improve rate determinations and the frequency dependence of FRBs.

The comparison of survey predictions with achieved discovery rate of pulsars and transients from the GHRSS survey is discussed in Section 8 .

\section{Survey processing}

To analyse the survey data, we used the 1456 core Hydrus cluster (30 Tflops) of the Jodrell Bank pulsar group and 512 core IBM cluster (10 Tflops) at the NCRA. The full GHRSS survey will generate approximately $50 \mathrm{~TB}$ of data and so far we have collected about 17 TB data corresponding to $35 \%$ completion of the survey. To optimise the data processing (i.e. considering the data transfer time from the observatory to the University of Manchester) and maximise the usage of the available CPU cycles on the available computing 
clusters we analysed $50 \%$ of the data with Hydrus and the rest with the IBM cluster.

\subsection{RFI flagging}

Like most low frequency radio telescopes, the GMRT is affected by local RFI. Frequently observed RFI occurrences at the GMRT include broadband impulsive signals originated from spark discharges from power-lines, narrow spectral impurity and short time-domain bursts. We performed zero-DM subtraction (Eatough et al. 2009) to remove broad band interference. The zero-DM subtraction procedure significantly reduced the number of spurious candidates detected in periodicity searches and in single pulse searches. This dramatically improved the detection significance towards broad band impulsive signals coming from pulsars. Figure 2 is the integrated profile and phasogram of PSR B1007-47 before and after zero-DM subtraction. Significant RFI can be seen as diagonal stripes caused by pulsed emission from power-lines or its subsequent harmonics, which are mitigated to a large extent after zero-DM subtraction and significant improvement of the pulsed detection is observed. Some residual RFI is still present in the data which can be seen in Figure 2. RFIFIND generally cleans up the data, especially for near to zero-DM residual RFIs, narrow-band spectral features and short time-domain bursts. We made this zero-DM subtraction routine a part of the publicly available SIGPROC distribution. Fourier domain analysis of the time series dedispersed at a $\mathrm{DM}=0$ enabled us to excise the powerline frequency, $50 \mathrm{~Hz}$. Finally, we employed PRESTO 19 -based RFIFIND package on the zero-DM subtracted time series to remove any remaining bursts of interference or strong spectral features. It is noteworthy that without these RFI flagging steps, $90 \%$ of the discovered pulsars reported in this paper would not have been detected.

\subsection{Dedispersion}

We dedispersed the data over a range of DMs, 0 to $500 \mathrm{pc} \mathrm{cm}^{-3}$, resulting in about 6000 dispersion trials for HGL and 10000 for MGL. The dispersion plan is generated following the DDPLAN of PRESTO in order to minimise the loss of sensitivity caused by dispersion smearing over a single frequency channel (Table 4). We reduced the time resolution for higher dispersion measure trials by down-sampling the data. For the GHRSS observing mode with 2048 frequency channels, the dispersive smearing within a frequency channel for

\footnotetext{
${ }^{19}$ http://www.cv.nrao.edu/ sransom/presto/
} 
a DM of $500 \mathrm{pc} \mathrm{cm}^{-3}$ is $1.9 \mathrm{~ms}$ at $322 \mathrm{MHz}$. Figure 3 shows the scatter broadening (Bhat et al. 2004) and dispersion smearing for the GHRSS survey as a function of DM. This indicates that with the newly implemented high resolution mode, the GHRSS survey sensitivity is not significantly affected by the instrumental broadening resulting from dispersion smearing up to a DM of $75 \mathrm{pc} \mathrm{cm}^{-3}$.

\subsection{Periodicity search}

The dedispersed time series were Fourier transformed and candidates with periodicities of known RFI sources were removed. We used a Fourier-based acceleration search method using the standard pulsar search techniques implemented in PRESTO. The acceleration search spanned a broadening of the Fourier peaks by up to 50 Fourier frequency bins (i.e. $z_{\max }$ of 50). This translates to $5 \mathrm{~m} \mathrm{~s}^{-2}$ line-of-sight acceleration for a $2 \mathrm{~ms}$ pulsar over $15 \mathrm{mins}$ of observing scan detected in all 8 harmonics. The search for periodicity was done using harmonic summing (up to 8 harmonics). The candidates for each DM were stored for comparison.

\subsection{Candidate sorting}

Candidates within a Fourier bin of another candidate were considered to be the same. We also ignored the candidates with summed incoherent power less than $4 \sigma$. Harmonically related candidates were removed, keeping the one with highest detection significance. We considered only those candidates that appear in at least three consecutive DMs to trace the DM dependence of signal-to-noise (SNR). A list of candidates was prepared after sorting.

\subsection{Candidate folding}

The zero-DM subtracted filter-bank data were dedispersed and folded with the periodicity parameters of sorted candidates in 32 sub-integrations and 64 frequency bands using PRESTO tool PREPFOLD. The left panel of Figure 4 shows an example of typical diagnostic plot from candidate folding for an MSP discovered in the GHRSS survey. 


\subsection{Candidate investigation}

A survey such as the GHRSS produces a large number of pulsar candidates, and it takes a lot of human effort to visually inspect the candidates in a reasonable amount of time. For example, a typical GHRSS survey scan with good RFI conditions produces around 100 to 200 folded candidates which are then manually investigated for possible pulsed emission. However, in the case of a moderately bad RFI environment, the number of candidates can be a few thousand for each pointing. We employed a neural network based binary classifier capable of separating candidates arising from noise or RFI, from those generated by radio pulsars following Lyon et al. (2015). We used this scoring method and machine learning algorithm to filter the best candidates for further inspection. The classifier is a very-fastdecision-tree and it uses the statistics (mean, standard deviation, kurtosis and skew) of the candidate profile and how the signal-to-noise varies as a function of DM as input scores. The classifier is applied to all periodicity candidates to label those as positive or negative, and only the positive candidates are viewed by eye. For example, 7700 candidates from one epoch were reduced to just 156 positive predictions, resulting in about 50 times reduction in the number of candidates to be viewed. Out of these positive predictions, 17 were re-detections of known pulsars or their harmonics, and 5 were promising pulsar candidates for follow up. We also examined all of the same 7700 candidates by eye to confirm that the classifier had performed accurately. The current version of the classifier was trained using a LOFAR data set but will be re-trained using the GHRSS data. The training set will include re-detections of known pulsars and discoveries from the GHRSS survey, as well as a sample of noise and RFI candidates from this survey. This will improve the performance of the classifier and reduce the number of false positives.

\section{7. $\quad$ Single pulse processing}

Besides the Fourier based periodicity searches, we performed a single pulse search to detect transient events, such as the RRATs or FRBs with possible extragalactic origin. We developed a PRESTO-based single pulse search pipeline for the Hydrus cluster of the Jodrell Bank pulsar group and IBM cluster at the NCRA for analysing the GHRSS survey data. After filter-bank conversion, zero-DM subtraction and RFI flagging, we dedispersed the raw data file at various trial DM values up to a DM of $2000 \mathrm{pc} \mathrm{cm}{ }^{-3}$ following DDPLAN. In order to accelerate the single pulse search we reduced the nominal time resolution to $1 \mathrm{~ms}$ and the dedispersed time series is further down sampled according to DDPLAN. We then searched for single pulses in the time series using the PRESTO code single_pulse_search.py (McLaughlin et al. 2006) above a detection significance of $5 \sigma$. Then we investigated the diagnostic plots. 
Figure 5 shows output from our CPU based single pulse search pipeline for the known pulsar J1559-4438. For a detected dispersed event we further investigate the dynamic spectrum to confirm the expected quadratic time delay nature of an astrophysical signal.

\subsection{GPU-based processing}

Since analysis of our data were limited by available compute power, re-processing the data in the future as we upgrade our compute power will possibly result in additional discoveries. We are in the process of setting up a multi-node Graphics Processing Unit (GPU) cluster, consisting of 5-nodes each with 4 Nvidia GTX 980 GPU ${ }^{20}$ each, at the University of Manchester to aid the GHRSS survey. Re-analysis of the GHRSS data in search for highly accelerated pulsars over a linear drift range of \pm 250 frequency bins is being performed with a variant of the PEASOU ${ }^{21}$ software implemented by us for the GHRSS survey. In addition, a GPU based single pulse search pipeline based on HEIMDALL (Barsdell et al. 2012) software was optimised and implemented for the GHRSS survey. This integrated multi GPU pipeline for pulsar and transient search implemented for the GHRSS survey is called BIFORST (paper in preparation). Figure 6 shows the output from this pipeline for single pulse searches.

\section{Survey sensitivity}

A pulsar is detectable (with $5 \sigma$ detection significance) in a survey with the incoherent array if it exceeds some minimum flux density $\left(S_{\text {pulsar }}\right)$ that can be calculated using the radiometer equation:

$$
S_{\text {pulsar }} \sim 5 \frac{T_{\text {rec }}+T_{\text {sky }}}{G \sqrt{B N_{\mathrm{p}} N_{\mathrm{a}} t}} \sqrt{\frac{w}{P-w}}
$$

where $T_{\text {rec }}$ and $T_{\text {sky }}$ are the temperatures of the receiver and sky respectively, $G$ the gain of individual antennas, $B$ the bandwidth, $N_{\mathrm{p}}$ the number of polarizations, $N_{\mathrm{a}}$ the number of antennas, $t$ the integration time, $w$ the effective pulse width (including all instrumental smearing), and $P$ the pulse period. As the telescope gain compensates for the larger bandwidths available at high frequencies $\left(\sim G_{\text {low }} / G_{\text {high }}>\sqrt{B_{\text {high }} / B_{\text {low }}}\right)$, it makes more sense to conduct surveys at lower frequencies as the larger beam-width greatly increases the sky coverage $\left(\propto \nu^{-2}\right)$ for a given dwell time $(t)$ with similar sensitivity.

\footnotetext{
${ }^{20} \mathrm{http}$ // /www.geforce.co.uk/hardware/desktop-gpus/geforce-gtx-980/specifications

${ }^{21}$ https://github.com/ewanbarr/peasoup
} 


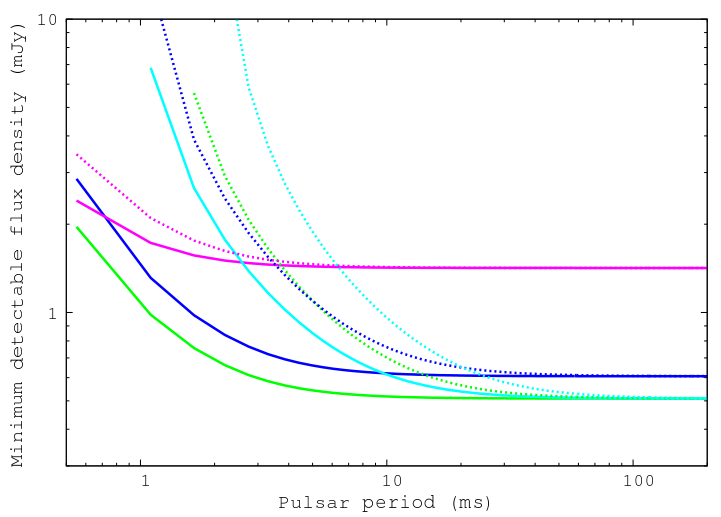

(a)

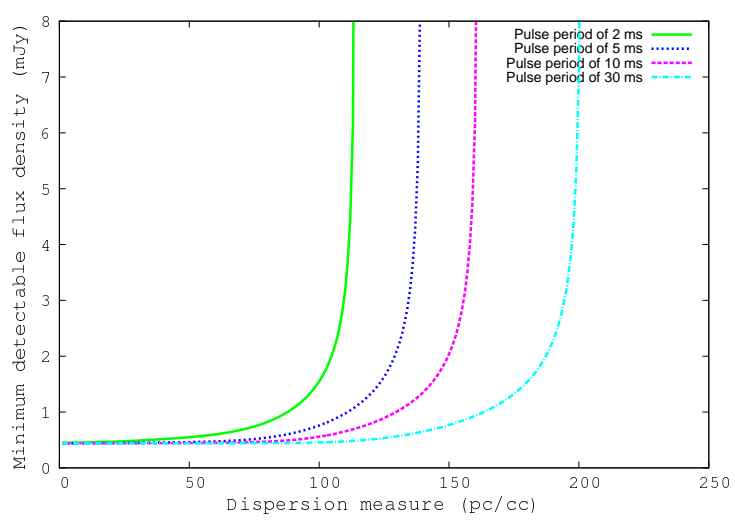

(b)

Fig. 7.- (a) Estimated sensitivity of the GHRSS (green), GBNCC (blue), HTRU (magenta) and GMRT-FERMI (cyan) surveys. The solid lines are for DM $50 \mathrm{pc} \mathrm{cm}^{-3}$ and dashed lines for $100 \mathrm{pc} \mathrm{cm}^{-3}$. Sensitivities are scaled to $322 \mathrm{MHz}$ with a spectral index of -1.7 . (b) Estimated sensitivity of the GHRSS survey to MSPs as a function of DM for a range of pulse periods, $2 \mathrm{~ms}$ (green), $5 \mathrm{~ms}$ (blue), $10 \mathrm{~ms}$ (magenta) and $30 \mathrm{~ms}$ (cyan).

For the GHRSS survey we calculate a theoretical survey sensitivity of $\sim 0.5 \mathrm{mJy}$ at $322 \mathrm{MHz}$ (considering the radiometer equation) for a $5 \sigma$ detection for $10 \%$ duty cycle, with the GMRT incoherent array of gain $2.5 \mathrm{~K} / \mathrm{Jy}\left(\sim G \sqrt{N_{\mathrm{p}} N_{\mathrm{a}}}\right)$ for $32 \mathrm{MHz}$ bandwidth and considering a system temperature of $106 \mathrm{~K}$. Figure $7 \mathrm{a}$ plots the estimated sensitivity of the GHRSS survey compared with GBNCC at GBT and HTRU at Parkes and the Fermidirected survey at GMRT at DMs of 50 and $100 \mathrm{pc} \mathrm{cm}^{-3}$. Figure 7a illustrates that the GHRSS survey is the most sensitive survey in the GBNCC complementary sky. Figure 7b plots the estimated sensitivity of the GHRSS survey for MSPs as a function of DM. This indicates that for a $5 \mathrm{~ms}$ pulsar even at a DM of $100 \mathrm{pc} \mathrm{cm}^{-3}$ the estimated sensitivity is $\sim 0.75 \mathrm{mJy}$, which is only 1.5 times the predicted GHRSS sensitivity. Enhanced time and frequency resolution of GHRSS survey by a factor of 4 (Section 2) enables an increased search sensitivity for MSPs up to a factor of 3 compared to the Fermi-directed MSP survey at the GMRT, which used $512 \times 0.0651 \mathrm{MHz}$ channels sampled at $61.44 \mu \mathrm{s}$. This is validated by observing J0248+4230 in these observing modes (Figure 8). With the newly implemented high resolution mode, the GHRSS survey is sensitive enough to detect MSPs at higher DMs as dispersion smearing is less than scatter broadening up to DM of $75 \mathrm{pc} \mathrm{cm}^{-3}$ (Figure 3 ). This will be useful considering $60 \%$ of the survey region will have $\mathrm{DM}<100 \mathrm{pc} \mathrm{cm}^{-3}$ based on Cordes et al. (2002). Thus the GHRSS survey modes are optimal for the targeted survey 
region. Figure $7 \mathrm{a}$ also illustrates that the GHRSS survey is the most sensitive survey in the GBNCC complementary sky. Table 2 shows a comparison between the major off-Galactic plane surveys.

A transient event of $5 \mathrm{~ms}$ duration will be detected at $10 \sigma$ detection significance if it exceeds some minimum flux density $\left(S_{\text {transient }}\right)$ calculated from the radiometer equation:

$$
S_{\text {transient }} \sim 10 \frac{T_{\text {rec }}+T_{\text {sky }}}{G \sqrt{B N_{\mathrm{p}} N_{\mathrm{a}} \tau}}
$$

where $\tau$ is the duration of the burst, $T_{\text {rec }}$ is $66 \mathrm{~K}$ and $T_{\text {sky }}$ is $40 \mathrm{~K}$. For the GHRSS survey we calculate a sensitivity of $1.6 \mathrm{Jy}$ for a $10 \sigma$ detection limit for $5 \mathrm{~ms}$ transient millisecond bursts, considering weak scattering (Thornton et al. 2013).

\section{Re-detection of known pulsars}

Re-detection of known pulsars and comparison with the catalogued flux density helps us to evaluate the survey sensitivity. The pulsars detected within the measurable primary beam gain pattern (i.e. up to the first null at $\sim \pm 100^{\prime}$ ) of the GMRT antenna ${ }^{22}$ were considered for flux density measurement. We had chosen only the highest signal-to-noise re-detection of a given pulsar, as some pulsars were re-detected in multiple adjacent scans. With $35 \%$ of the GHRSS survey complete, we had 23 such re-detections of known pulsars. There are 30 known pulsars above $15 \sigma$ detection significance present within the observed GHRSS survey pointings. Most of the non detections of known pulsars correspond to the pulsars being at the edge of beam and/or presence of RFI. We exploited the simultaneous availability of visibilities, to determine calibrated interferometric flux densities. Imaging was done using a pipeline that was developed specifically for analysis of GMRT data (Kudale et al., in preparation). The raw interferometric data were converted to FITS and then calibrated and flagged using the FLAGCAL pipeline (Prasad et al. 2012; Chengalur et al. 2013). We performed flux calibrations using flux densities of the observed phase calibrators as given in the VLA calibrator manua 23 . The data were then imaged using a custom imaging pipeline built around utilities in the CASA package as well as the source identification package PYBDSM (paper in preparation). PYBDSM was used to identify regions with emission in the dirty image which were set as the CLEAN boxes in the cleaning and self-calibration cycles. First a wide, low resolution image was made (using mainly data from the central square

\footnotetext{
${ }^{22}$ http://www.ncra.tifr.res.in:8081/ñgk/primarybeam/beam.html

${ }^{23}$ https://science.nrao.edu/facilities/vla/docs/manuals/cal
} 
antennas). We used this to identify sources that lie outside the half-power beam-width (HPBW) of the telescope. Then a high resolution image using all available antennas was made. The image covers the HPBW, but with extra "flanking fields" centred on all of the outlying sources identified in the low resolution image. The imaging and self calibration procedures typically included two rounds of phase-only self calibration followed by a final round of phase and amplitude calibration. Since most of the sources have been observed at very low elevation angles (as the fields in this survey were all southern fields) refractive effects can cause a significant shift in the position of the sources. Wherever possible this shift was calibrated using catalogued observations at higher frequencies (e.g. using the 843 $\mathrm{MHz}$ SUMSS24 catalogue in fields that overlap with that survey, or sources with known positions in the $\mathrm{NED}^{25}$ database). After this correction we searched for emission around the known position of the pulsar. The measured flux density was then corrected for the primary beam attenuation to yield the final flux density of the pulsar. We could determine an interferometric flux density for 12 such re-detected fields having overlap with SUMSS/NED catalogued observations. Other re-detections could not be detected in the image due to increased noise near the pulsar position and/or significant flagging during imaging. Figure 9 shows the re-detection of PSR J1312-5402 at 49' offset from the survey pointing centre, which clearly illustrates that pulsars of a few mJy can even be detected outside the HPBW of the survey pointing. Table 6 lists the offset from the survey pointing centre, pulsed-SNR with primary beam correction and the corresponding imaging flux density of the re-detected pulsars. Similar to the GBNCC survey (Stovall et al. 2014) we estimated the expected catalogued flux density at $322 \mathrm{MHz}$ for each of these pulsars, either by using the spectral index when available in the ATNF catalogue, or by extrapolating $400 \mathrm{MHz}$ mean flux density reported in the ATNF catalogue assuming a spectral index of -1.7 . Large uncertainties could be associated with the catalogued $400 \mathrm{MHz}$ flux densities as well with the assumption of -1.7 spectral index, which indicates that the expected flux density estimates at 322 $\mathrm{MHz}$ have low accuracy. Figure 10 compares the observed interferometric flux density with the extrapolated catalogued flux density. The majority of the detections have observed flux densities within $\pm 50 \%$ of the expected values (marked by green shaded region). In conjunction to the uncertainties involved in estimating the expected catalogued flux density, the observed sensitivity degradation for some of the re-detections could also be caused due to scintillation or significant flagging due to RFI.

\footnotetext{
${ }^{24}$ http://heasarc.gsfc.nasa.gov/W3Browse/radio-catalog/sumss.html

${ }^{25}$ https://ned.ipac.caltech.edu
} 


\section{Discoveries}

In this paper we report pulsar and transient search results from $35 \%$ completion of the GHRSS survey covering $1000 \mathrm{deg}^{2}$ of sky. With the periodicity search we discovered 10 pulsars including one millisecond pulsar in a binary system. Figure 11 shows the discovery profiles and Table 5 lists the periods, dispersion measures and discovery flux density values. Though some of the newly discovered pulsars are reasonably strong and might have been missed by previous surveys (e.g. J0418-4154, J0702-4956 and J0514-4407), we also discovered a few weak pulsars, indicating the highly sensitive nature of the survey even in the presence of some residual RFI after flagging. At the discovery epoch the newly discovered MSP had an estimated flux density of $1.6 \mathrm{mJy}$ which is close to the theoretical sensitivity considering the system temperature of $109 \mathrm{~K}$ (Haslam et al. 1982) and 30\% duty cycle. We confirmed all the discoveries in subsequent epochs of observations. The GHRSS discoveries have dispersion measures ranging from $15.4 \mathrm{pc} \mathrm{cm}^{-3}$ (for PSR J0514-4407) up to $133 \mathrm{pc} \mathrm{cm}^{-3}$ (for PSR J1456-48) and periods from $5.04 \mathrm{~ms}$ (for PSR J2144-5237) up to $1169.89 \mathrm{~ms}$ (for PSR J1559-44). Most of the newly discovered pulsars (e.g. J0418-4154, J0702-4956, J0919-42, J1255-46, J1456-48, J1559-44 and J1708-52) have single peaked profiles. PSR J1947-43 has a relatively wide profile and there is a hint of a double peaked profile. For PSR J0514-4407 a double component pulse profile is observed. The profile shapes were confirmed with multi-epoch observations. The $52.04 \mathrm{~ms}$ rotational period of PSR J1255-46 puts it in the domain of young pulsars or double neutron star relativistic binaries. A more detailed study of this pulsar is in preparation. The $5.04 \mathrm{~ms}$ MSP J2144-5237 has a multi-component profile. The right panel of Figure 4 shows the sensitive coherent array observations aided by precise localisation (Section 9). The flux density of this MSP varies dramatically due to scintillation, indicating the need for re-observations of candidate MSPs with longer integrations. A drift over Fourier frequency by $4 \times 10^{-4} \mathrm{~Hz}$, which corresponds to a period derivative of $-1.4 \times 10^{-12} \mathrm{~s} \mathrm{~s}^{-1}$ or a line-of-sight acceleration of $1.4 \mathrm{~m} \mathrm{~s}^{-2}$ observed for J2144-5237 over two hours, indicates that this MSP is in binary system. PSR J2144-5237 could be a good timer for inclusion in the International Pulsar Timing Array.

The area normalised discovery rate of the GHRSS survey is 0.01 pulsars per deg $^{2}$ which is higher than the 0.006 pulsars per $\mathrm{deg}^{2}$ achieved with the GBNCC survey (Section 4). It may be that this higher discovery rate is purely due to low number statistics at this stage or could perhaps represent an underestimate of the sensitivity of the GMRT in the incoherent mode or perhaps an overestimate of the GBNCC sensitivity. It may be useful to survey an overlapping region of sky to enable a more direct comparison between these low frequency surveys. However the GHRSS pulsar discovery rate is lower than the prediction by PSRPOPPY prediction (Section 4) like many of the other surveys. 
We have not detected any FRB or RRAT with the single pulse processing in the GHRSS survey. However, considering the FRB rate predicted in Section 4 we should have detected only about $2_{-1}^{+1}$ FRBs in this survey region based on a flat spectral index consideration. Absence of which is not constraining enough considering factor of few lower FRB prediction by recent studies (Rane et al. 2015; Champion et al. 2015; Petroff et al. 2014).

\section{Localisation of the GHRSS discoveries}

We utilised the interferometric imaging capability of the GMRT to localise pulsars and transient events. Such localisation in the image plane is very important for FRBs for host galaxy identification and multiwavelength follow up.

\subsection{Localisation of the pulsars with gated correlator}

A gating interferometer is an excellent tool to improve the noise statistics in order to increase the detection significance towards a pulsed signal. The development of a coherently dedispersed gated correlator for the GMRT is detailed in Roy et al. (2013). In this design, the visibility time-series were derived off-line from the recorded raw voltage data and were binned using multiple gates and on each gate they were folded using the best-fit topocentric rotational model, derived from the synchronously generated incoherent beam data. We improved the noise statistics further by subtracting the visibilities of adjacent gates. The ON-OFF subtracted visibilities were then calibrated and flagged using FLAGCAL pipeline (Prasad et al. 2012; Chengalur et al. 2013). The ON-OFF image was made using the AIPS package, where the pulsar is unambiguously localised as a point source. For pulsars with $10 \%$ duty cycle, we expect to get a factor of 3 improvement in detection significance in the gated image plane compared to the continuum plane. The positional uncertainty therefore reduces from $80^{\prime}$ (half-power beam-width at $322 \mathrm{MHz}$ ) to $<20^{\prime \prime}$ (typical synthesized beam used in the image made at $322 \mathrm{MHz}$ ).

We recently started follow up of these pulsars using the coherently dedispersed gated correlator which resulted in the successful localisation of 4 pulsars, namely: J0418-4154, J0702-4956, J0514-4407 and J2144-5237 with an accuracy of $<10^{\prime \prime}$ (positions are listed in Table 7). Figure 12 plots the ON-OFF gated images of these 4 pulsars. Such timing independent, rapidly determined and fairly precise positions allow us to use sensitive coherent beams (up to $5 \times$ the sensitivity of the incoherent beam) of the GMRT for follow up timing 
observations, substantially reducing the use of telescope time. Further improvement of the detection significance is seen while using the coherent array, as it is better resistant to RFI. Right panel of Figure 4 shows the huge improvement in sensitivity achieved with coherent array observations for PSR J2144-5237. Moreover, such a priori astrometric models allow, rapid convergence in pulsar timing as discussed in Section 10.

\subsection{Localisation of FRBs in the image plane}

In addition to localisation of the pulsars, simultaneous availability of the visibility data during the high resolution survey provides us the opportunity to localise the transient events detected in time-domain searches. All 17 known FRBs have been discovered with single dish telescopes and interferometric detection of an FRB is of significant importance. This technique is successfully utilised in the study of the eclipsing pulsars with the GMRT (e.g. PSR J1227-4853 (Roy et al. 2015)). An example of interferometric localisation of a redetection of a known pulsar in the GHRSS survey (Figure 9) also illustrates the pipeline that will be followed for localisation of possible transient detection in the GHRSS survey. Assuming that interferometric gain is similar to coherent array gain (i.e. 5 times of incoherent array), FRBs with flux density $>3$ Jy could be localised at $>5 \sigma$ significance in the image plane from simultaneously recorded visibilities at $2 \mathrm{~s}$ resolution.

\section{Timing and follow up of the GHRSS discoveries}

We are continuing regular timing follow up of the GHRSS pulsars, with the coherent array of the GMRT, to determine their rotational and binary parameters (for the MSP) and place them on the $P-\dot{P}$ diagram. Long term timing will also allow us to probe the possible optical and $\gamma$-ray counterparts of the newly discovered pulsars. The timing-independent rapid astrometric measurement of newly discovered pulsars using gated imaging helps to break the degeneracy in the timing fit caused by co-variance between pulsar position and rotational parameters. We successfully demonstrated this by timing two of the localised pulsars, J0418-4154 and J0702-4956, using sparsely spaced TOAs over a span of $\sim 200$ days. Considering reasonable fractional bandwidth of $10 \%$ at $322 \mathrm{MHz}$, we determined the DM using timing fits of sub-band TOAs. The timing ephemeris of both the pulsars are given in Table 8. The post-fit residuals are shown in Figure 13. Residuals for J0702-4956 are fitted with three timing models having different astrometric positions: the gated interferometric position, $1^{\prime}$ offset and $5^{\prime}$ offset from the gated interferometric position, which is illustrated in Figure 14. The residuals increase as the position offset increases and the residuals are 
wiggled in phase at $5^{\prime}$ offset. This warrants more closely spaced and regularly sampled TOAs to overcome the phase ambiguity caused by position error. Thus a priori knowledge of a pulsar position from the gated-image is extremely important, considering that the discovery position can be anywhere within the HPBW $\left( \pm 40^{\prime}\right)$ or even outside the HPBW (e.g. Figure 9 .

Following the localisation of a GHRSS pulsar we routinely check for nearby sources from existing optica ${ }^{26}$ and high-energy catalogue ${ }^{27}$. For one of the GHRSS discovery pulsar, J0514-4407, we found a possible counterpart 3FGL J0514.6-4406, with a position uncertainty of about $0.16^{\circ}$. This is only $1.8^{\prime}$ from the pulsar, so well within predicted $\gamma$-ray position. However, none of the other pulsars listed in Table 7 have 2FGL/3FGL counterparts. We plan to fold the Fermi photons with the timing model of the newly discovered pulsars to check if any of these pulsars exhibit $\gamma$-ray pulsations. This will provide input towards the radio-loud versus radio-quiet $\gamma$-ray pulsar ratio, which is a reasonable differentiator between $\gamma$-ray population models.

\section{Relevance to the SKA}

Discoveries of pulsars, in particular MSPs, are hindered by their radio faintness that demands deeper searches with larger telescopes and we are reaching the limit of what is possible for fully steerable single dishes. Large arrays of many smaller telescopes are the future for large radio telescopes leading ultimately to the world's largest telescope the SKA. The SKA's wide field-of-view, high sensitivity, multi-beaming and sub-arraying capabilities, coupled with advanced pulsar search backends, will result in the discovery of a large population of pulsars (Keane et al. 2015). As it is currently the largest telescope in the metre-wavelength regime, the GMRT, an SKA pathfinder telescope, is the prototype for the SKA in many ways, and provides an excellent test bed for new techniques. Tools for efficient RFI mitigation, optimised search techniques, synchronous beam-formation and imaging will provide vital input to the SKA. In particular the high resolution GHRSS data are being used for testing the optimised dedispersion and de-acceleration plan of the pulsar search sub-element (PSS) of SKA. The periodicity search for the GHRSS needs an 8 million point transform, similar to the requirement of the SKA. Moreover, the rapid convergence in pulsar timing model possible with precise localisation of the pulsars with gated imaging described in Section 9 and 10, demonstrates a feature that may be applicable in the SKA.

\footnotetext{
${ }^{26}$ http://simbad.u-strasbg.fr/simbad/sim-fcoo

${ }^{27}$ http://fermi.gsfc.nasa.gov/ssc/data/access/lat/4yr_catalog/
} 
The GHRSS survey is at a frequency that overlaps with the SKA1 low frequency instrument (SKA1-Low) and considering SKA1-Low will have sensitivity $\sim 0.05 \mathrm{mJy}$ at $350 \mathrm{MHz}$ (Keane et al. 2015) (i.e. 10 times GHRSS sensitivity) all the pulsars discovered with the GHRSS survey will be able to be timed and studied with SKA1-Low, providing an excellent opportunity to prepare for searches and timing for this next generation telescope. The GPU based processing pipeline implemented by us, BIFORST, performs a time-domain acceleration search on pulsar survey data, and the processing steps roughly follow those of the PSS timedomain processing pipeline. The GHRSS survey data are concurrently being processed by time-domain acceleration search using GPU and frequency-domain acceleration search using CPU, and thus provides detailed comparison between the algorithms, which can be a vital input to SKA choices to decide the appropriate search methodology. These results will provide very valuable input into directing the prototyping and design effort for pre-construction stage. In future for the GHRSS survey we plan to use the baseband recording facility of the GMRT Software Backend followed by multi-pixel coherent beamformation (Roy et al. 2012). Pixelisation of the full field-of-view while scanning the extreme southern sky with the GMRT will provide $3 \times$ sensitivity improvement and $20 \times$ increase in compute cost. For the extreme southern sky we will require 300 beams which is $\sim 10 \%$ compute cost of SKA pulsar search.

\section{Conclusion}

In this paper we described the system configuration and initial discoveries from the GHRSS survey. We described the high resolution observing modes developed for this survey and the data processing pipeline for periodicity and single pulse search. Our survey sensitivity is comparable to other ongoing low frequency surveys, and we are targeting a portion of sky which has not been surveyed at lower frequencies like $322 \mathrm{MHz}$ for the last two decades. With 35\% completion of the GHRSS survey covering $1000 \mathrm{deg}^{2}$ of sky we discovered 10 pulsars including $1 \mathrm{MSP}$ which corresponds to one of the best pulsar per $\mathrm{deg}^{2}$ discovery rates of any survey off the Galactic plane. We also re-detected 23 previously known pulsars with the expected detection significance, which were in-beam pulsars, not necessarily at the pointing centre. The simultaneous time-domain search and imaging capability of this survey provides an opportunity of discovery and localisation of pulsars and transients. Utilising this we localised 4 of the newly discovered pulsars in the gated image plane. With the aid of $\sim$ $10^{\prime \prime}$ localisation, we obtained the timing solutions of 2 of the discoveries with relatively short data spans $(<1 / 2$ year). In addition to the regular emission from pulsars, the GHRSS survey will reveal transient events like the RRATs or the FRBs. With an ongoing enhancement of processing power we expect more results soon. 
B. Bhattacharyya acknowledges support of Marie Curie grant PIIF-GA-2013-626533 of European Union. P. Ray's contributions to this work were supported by the Chief of Naval Research (CNR). We thank the reviewer of our paper for a detailed review that improved the paper significantly. We thank A. Holloway and R. Dickson for making the Hydrus computing cluster at University of Manchester available for this survey analysis. We also thank them in helping to set-up the GPU cluster. We also acknowledge the generous support of $\mathrm{V}$. Venkatasubramani, S. J. Bhachal and A. Meghe at the NCRA in keeping the IBM cluster running for data processing. We thank L. Levin for PSPOPPY related discussion and M. Mickaliger for plotfil display tool. We also thank E. Keane for a discussion on the paper. We acknowledge the help from N. Mohan for modifying the PYBDSM package to suit the imaging analysis of the GHRSS fields. We acknowledge support of GMRT operators. The GMRT is run by the National Centre for Radio Astrophysics of the Tata Institute of Fundamental

Research. The National Radio Astronomy Observatory is a facility of the National Science Foundation operated under cooperative agreement by Associated Universities, Inc.

\section{REFERENCES}

Archibald, A. M., Stairs, I. H., Ransom, S. M., et al. 2009, Science, 324, 1411.

Alexander Y. C., Andrei M. B., 2014, ApJL, 795, 22.

Antoniadis, J. et al. 2013, Sci, 340, 448.

Archibald, A. M. et al. 2009, Sci, 324, 1411.

Backer, D. C., 1970, Nature, 228, 1297.

Bailes, M., et al. 2011, Sci, 333, 1717.

Bailes, M., et al. 1994, ApJ, 425, 41.

Barsdell, B. R., Bailes, M., Barnes, D. G., \& Fluke, C. J. 2012, Astronomical Data Analysis Software and Systems XXI, 461, 37.

Burke-Spolaor S., Bannister K. W., 2014, ApJ, 792, 19

Barr et al., 2011, AIP conf. proceeding, 2011, 1357, 52.

Bates et al. 2014, MNRAS, 439, 2893.

Bhat et al. 2004, ApJ, 605, 759. 
Burgay et al. 2006, MNRAS, 368, 283.

Bhattacharyya et al., 2013, ApJ Letters, 772, 12.

Boyles J. et al., 2013, ApJ, 763, 80.

Camilo et al., 2006, Nature, 442, 892.

Chengalur, J. N., 2013, NCRA Technical Report, NCRA/COM/001.

Coenen et al., 2014, A\&A, 570, 60.

Cordes et al., 2006, ApJ, 637, 446.

Cordes J. M., Lazio T. J. W., 2002, preprint (arXiv:astro-ph/0207156).

Cordes J. M., Wasserman I., 2015, preprint (arXiv:1501.00753).

Champion D. J. et al. 2015 (submitted) preprint (arXiv:1511.07746).

Deneva J. et al. 2013, ApJ, 775, 51.

Deng W., Zhang B., 2014, ApJ, 783, L35.

Detweiler S. et al. 1979, ApJ, 234, 1100.

Haslam C. G. T. et al. 1982, A\&A, 47, 1.

Faucher-Giguere C. A., Kaspi V. M., 2006, ApJ, 643, 332.

Falcke H., Rezzolla L., 2014, A\&A, 562, 137.

Gil J., Melikidze G. I., Geppert U., 2003, A\&A, 407, 315.

Gupta, Y., Gothoskar, P., B., Joshi, B., C., Vivekanand, M., Swain, R., Sirothia, S., and Bhat, N., D., R., 2000, in IAU Colloq. 177, Pulsar Astronomy, ed. Kramer, M., Wex, N., and Wielebinski, R., (ASP Conf. Ser. 202, San Francisco: ASP), 277.

Keane et al., 2015, MNRAS, 409, 619.

Keane E. F., Petroff E., 2015, MNRAS, 447, 2852.

Keith et al., 2010, MNRAS, 409, 619.

Kramer M. et al., 2006, MNRAS, 312, 549.

Kulkarni S. R., Ofek E. O., Neill J. D., Zheng Z., Juric M., 2014, ApJ, 797, 70. 
Lazarus et al., 2013, IAU Symp. 291, Neutronstars and Pulsars: challenges and Opportunities after 80 years, ed. J. van Leeuwen (Cambridge: Cambridge Univ. Press), 237.

Lorimer et al., 2012, Proceedings IAU Symposium No. 291 (arXiv:12102746V1).

Lorimer et al., 2008, LRR, 11, 8.

Lorimer et al. 2007, Science, 318, 777.

Lorimer et al., 2006, MNRAS, 372, 777.

Lorimer, D. R. \& Kramer, M., 2004, Handbook of Pulsar Astronomy, Vol. 4. Cambridge, UK, 211.

Levin et al., 2013, MNRAS, 434, 1387.

Lee, K. J. et al., 2012, MNRAS Letters, 423,2642.

Lattimer, J. M., Prakash M. 2004, Science, 304, 536.

Lundgren S. C., Cordes J. M., Ulmer M., Matz S. M., Lomatch S., Foster R. S., Hankins T., 1995, ApJ, 453, 433.

Luan J., Goldreich P., 2014, ApJ, 785, L26.

Lyubarsky Y., 2014, MNRAS, 442, L9.

Lyne, A. et al., 2010, Science, 329, 408.

Lyne, A. et al., 2006, ASPC, 105, 73.

Lyon, R. J. et al., 2015, MNRAS (submitted).

Manchester et al., 2001, MNRAS, 328, 17.

McLaughlin M.A., Lyne A.G., Lorimer D.R., et al. 2006, Nature, 439, 817.

Mottez F., Zarka P., 2014, A\&A, 569, A86.

Petroff, E. et al. 2014, MNRAS, 789, 26.

Prasad, J., Chengalur, J. N. 2012, Experimental Astronomy, 33, 157.

Ransom, S., et al. 2014, Nature, 505, 520.

Rane, A., Lorimer D. R., Bates S. D. et al. 2015, MNRAS (submitted), arXiv:1505.00834. 
Roberts, M. S. E., 2011, AIP Conf. Proc. 1357, Radio Pulsars: An Astrophysical Key to Unlock the Secrets of the Universe ed. M. Burgay, N. DAmico, P. Esposito, A. Pellizzoni, \& A. Possenti (Melville, NY: AIP), 127.

Ray, P. S. et al., 2012, 2011 Fermi Symposium proceedings, arXiv:1205.3089.

Roy J., Gupta Y., Ue-Li Pen, Peterson J.B., Kudale S., Kodilkar J., 2010, Experimental Astronomy, 28, 55.

Roy J. et al., 2012, MNRASL, 427, 90.

Roy J. \& Bhattacharyya B., 2013, ApJL, 765, 45.

Roy J., 2013, PhD Thesis.

Roy J. et al., 2015, ApJL, 800, 12.

Ruderman, M.A. \& Sutherland, P.G., 1975, ApJ, 196, 51.

Spitler L. G. et al., 2014, ApJ, 790, 101

Stovall et al., 2014, ApJ, 2014, 791, 67.

Swarup, G., Ananthakrishnan, S., Subrahmanya, C., R., Rao, A., P., Kulkarni, V., K., and Kapahi, V., K., 1997, in High Sensitivity Radio Astronomy, ed. Jackson, N., and Davis, R., J., (Cambridge: Cambridge University Press), 217.

Thornton D. et al., 2013, Science, 341, 53 
Table 1: Survey parameters of the GHRSS survey for the MGL and HGL components

\begin{tabular}{|l|c|c|}
\hline Survey & $\begin{array}{c}\text { MGL } \\
\text { with HiRes1 }\end{array}$ & $\begin{array}{c}\text { HGL } \\
\text { with HiRes2 }\end{array}$ \\
\hline Galactic region & $5<|b|<20^{\circ}$ & $|b|>20^{\circ}$ \\
Declination & $-40^{\circ}<$ Dec $<-54^{\circ}$ & $-40^{\circ}<$ Dec $<-54^{\circ}$ \\
Integration time & $1200 \mathrm{~s}$ & $900 \mathrm{~s}$ \\
Sampling time & $61.44 \mu \mathrm{s}$ & $30.72 \mu \mathrm{s}$ \\
Bandwidth & $32 \mathrm{MHz}$ & $32 \mathrm{MHz}$ \\
Number of channels & 2048 & 1024 \\
Frequency Resolution & $15.625 \mathrm{kHz}$ & $31.25 \mathrm{kHz}$ \\
Number of pointings & 682 & 911 \\
Sky coverage & $1227 \mathrm{deg}^{2}$ & $1639 \mathrm{deg}^{2}$ \\
Data/pointing & $37 \mathrm{~GB}$ & $28 \mathrm{~GB}$ \\
Total data & $25 \mathrm{~TB}$ & $25 \mathrm{~TB}$ \\
\hline
\end{tabular}


Table 2: Major ongoing or recently completed off-Galactic plane surveys

\begin{tabular}{|c|c|c|c|c|}
\hline $\begin{array}{l}\text { Survey name } \\
\text { - Telescope }\end{array}$ & $\begin{array}{c}\text { Frequency } \\
\text { of search } \\
(\mathrm{MHz})\end{array}$ & Sky coverage & Discoveries & $\begin{array}{c}\text { Sensitivity }^{\dagger} \\
\text { (mJy) }\end{array}$ \\
\hline HTRU $^{1}$ & 1352 & $\begin{array}{c}-120^{\circ}<1,1<30^{\circ} \\
|b|<15^{\circ}\end{array}$ & 104 PSR, 26 MSP & 1.5 \\
\hline $\begin{array}{l}\text { - Parkes } \\
\text { HTRU-N } \\
\text { - Effelsberg }\end{array}$ & 1360 & $|b|>15^{\circ}$, Dec $>-20^{\circ}$ & 12 PSR & 1.5 \\
\hline $\begin{array}{l}\mathrm{GBNCC}^{2} \\
-\mathrm{GBT}^{2}\end{array}$ & 350 & $\begin{array}{l}\text { Dec }>-40^{\circ} \\
19500 \mathrm{deg}^{2}\end{array}$ & 108 PSR, 12 MSP & 0.6 \\
\hline $\begin{array}{c}\text { GBTdriftscan }^{3} \\
- \text { GBT }\end{array}$ & 350 & $-21^{\circ}<$ Dec $<26^{\circ}$ & $26 \mathrm{PSR}, 7 \mathrm{MSP}$ & 0.9 \\
\hline $\begin{array}{c}\text { AO } 327^{4} \\
- \text { Arecibo }\end{array}$ & 327 & $0^{\circ}<$ Dec $<28^{\circ}$ & $24 \mathrm{PSR}, 3 \mathrm{MSP}$ & 0.3 \\
\hline $\begin{array}{l}\text { LOTAAS }^{5} \\
- \text { LOFAR }\end{array}$ & 135 & $\begin{array}{l}\text { Dec }>0 \\
5156 \mathrm{deg}^{2}\end{array}$ & 18 PSR & 0.3 \\
\hline $\begin{array}{l}\text { GHRSS }^{\ddagger 6} \\
- \text { GMRT }^{-1}\end{array}$ & 322 & $\begin{array}{c}-20^{\circ}<\text { Dec }<-54^{\circ} \\
1000 \mathrm{deg}^{2}\end{array}$ & 10 PSR, 1 MSP & 0.5 \\
\hline
\end{tabular}

$\dagger$ : Sensitivity calculated at $322 \mathrm{MHz}$ considering $5 \sigma$ limit for $10 \%$ duty cycle for spectral index of -1.7

‡: Full GHRSS survey will cover $2866 \mathrm{deg}^{2}$.

1: http://researchbank.swinburne.edu.au/vital/access/manager/Repository/swin:31985

2: http://arcc.phys.utb.edu/gbncc/

3: http://astro.phys.wvu.edu/GBTdrift350/

4: http://www.naic.edu/deneva/drift-search/

5: http://www.astron.nl/lotaas

6. http://www.jb.man.ac.uk/research/pulsar/Resources/ghrss.html 
Table 3: Parameters for PSRPOPPY simulations

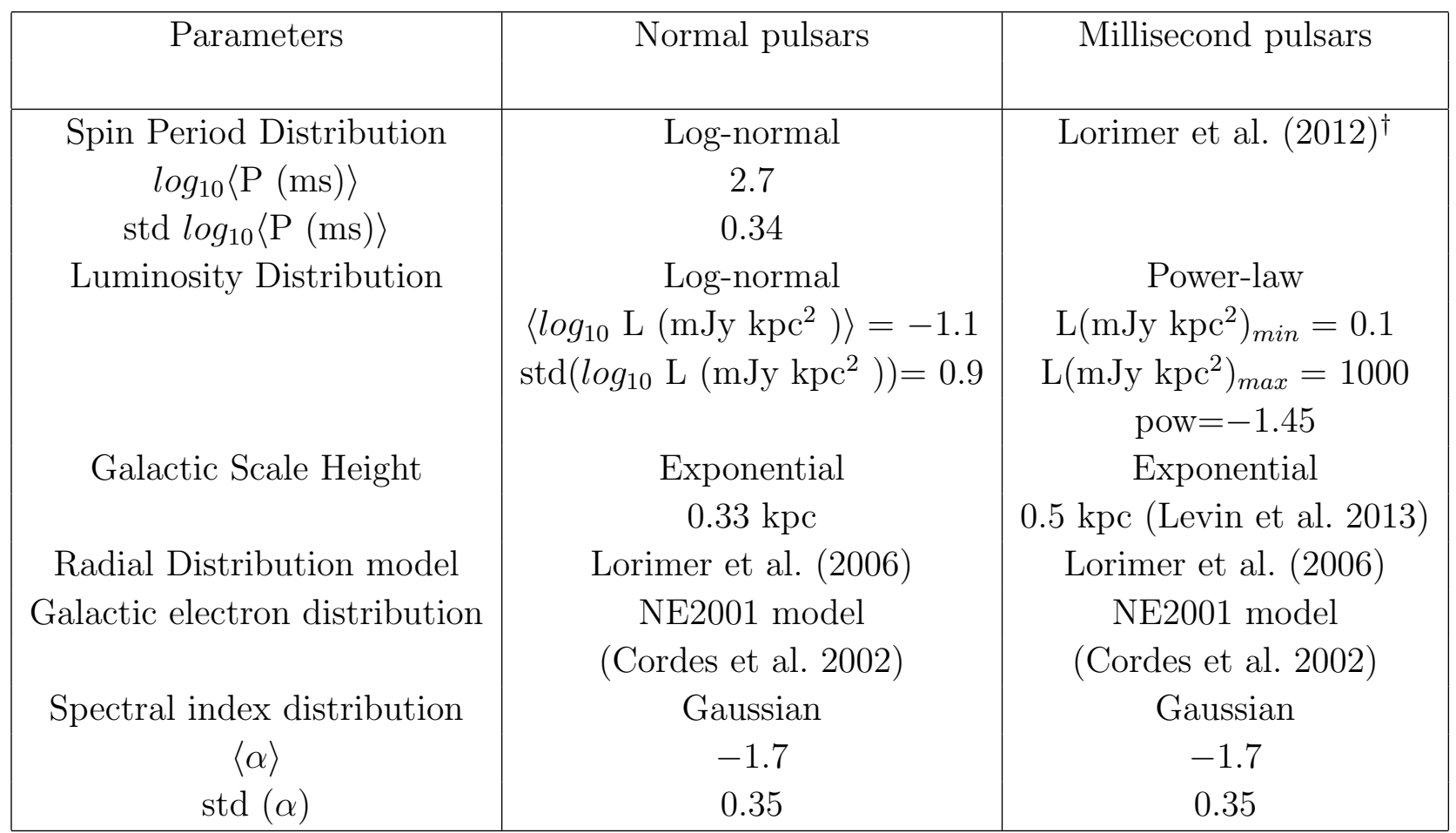

$\dagger$ : with this built-in option in PSRPOPOY, periods of millisecond pulsars are picked in at random from data base provided in Lorimer et al. (2012).

Table 4: Dispersion plan and corresponding time resolution for HGL and MGL according to the DDPLAN of PRESTO

\begin{tabular}{|l|c|c|c|c|}
\hline \multirow{2}{*}{ DM range } & \multicolumn{2}{|c|}{ HGL } & \multicolumn{2}{c|}{ MGL } \\
& DM step $\left(\mathrm{pc} \mathrm{cm}^{-3}\right)$ & Sampling resolution $(\mu \mathrm{s})$ & DM step & Sampling resolution $(\mu \mathrm{s})$ \\
\hline $1-25$ & 0.010 & 30.72 & 0.010 & 61.44 \\
$25-50$ & 0.025 & 61.44 & 0.010 & 61.44 \\
$50-100$ & 0.050 & 122.88 & 0.025 & 61.44 \\
$100-150$ & 0.100 & 245.76 & 0.050 & 122.88 \\
$150-300$ & 0.200 & 491.52 & 0.100 & 245.76 \\
$300-500$ & 0.500 & 491.52 & 0.200 & 491.52 \\
\hline
\end{tabular}



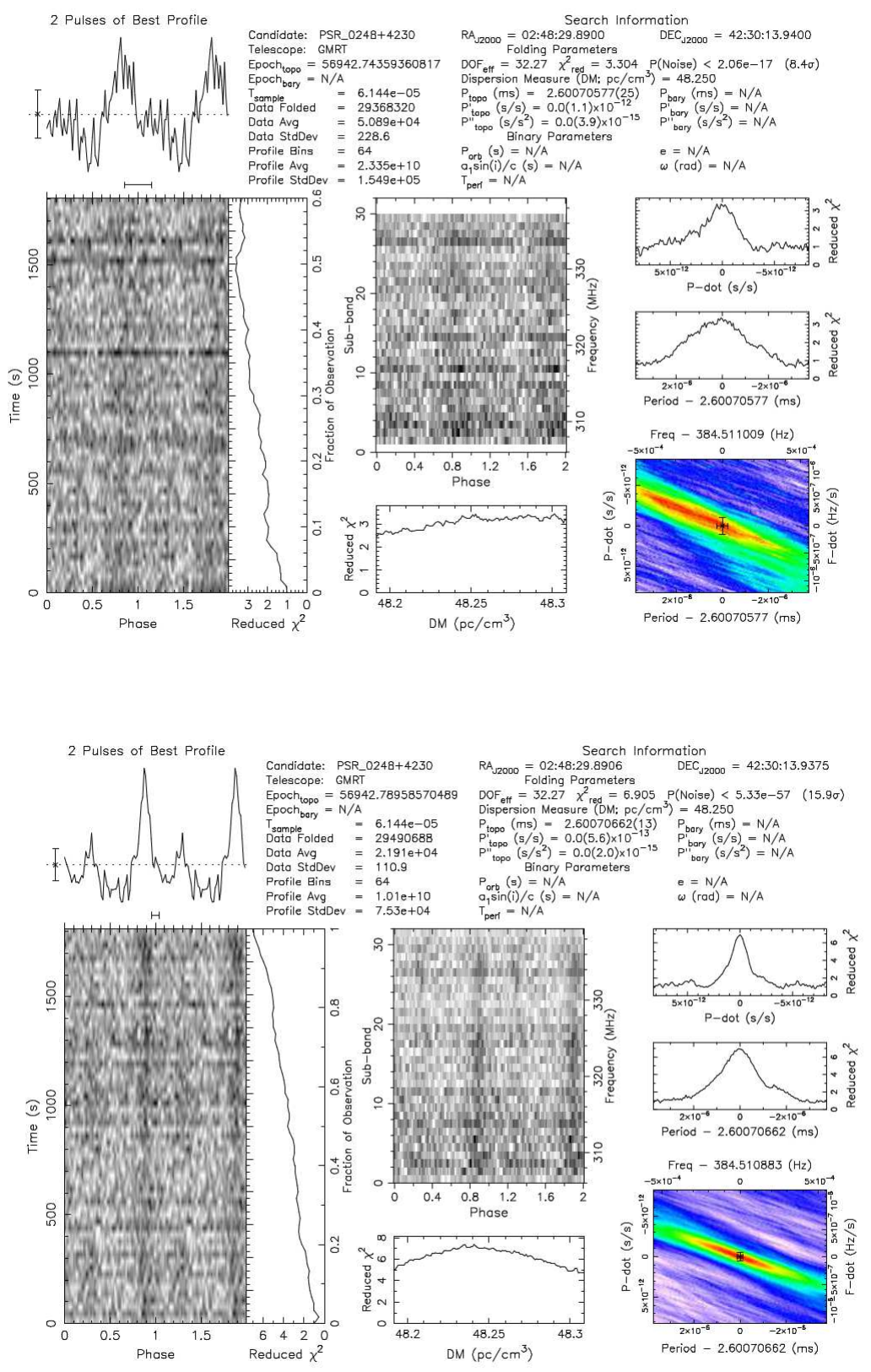

Fig. 8.- Output of PRESTO based GHRSS search pipeline for the MSP J0248+4230 to demonstrate the improvement in detection significance with the high resolution observing modes developed in the GHRSS survey. Top panel: with $32 \mathrm{MHz}$ observing band split into 512 frequency channels recorded every $61.44 \mu \mathrm{s}$, Bottom panel: with $32 \mathrm{MHz}$ observing band split into 2048 frequency channels recorded every $61.44 \mu \mathrm{s}$. 

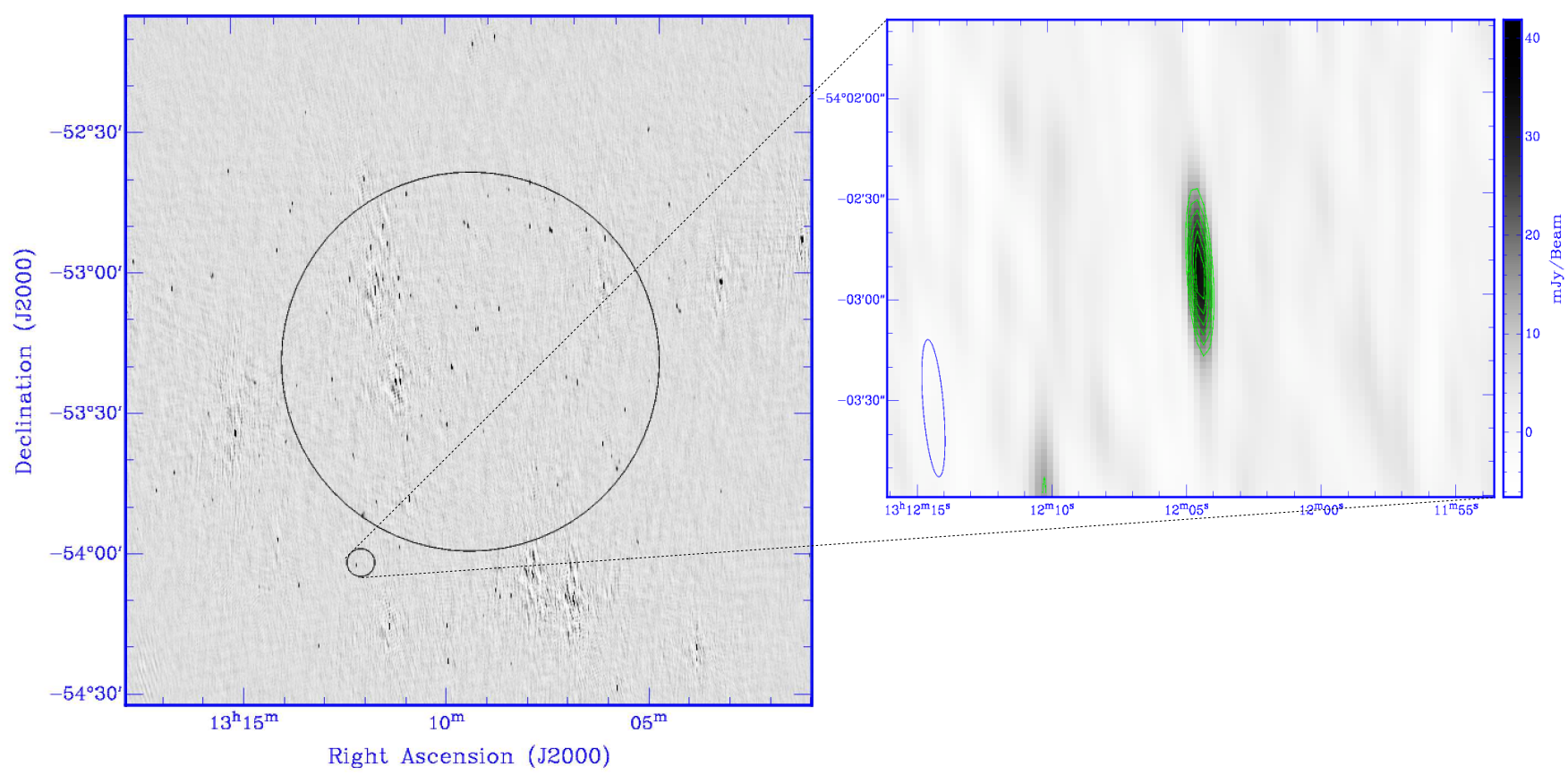

Fig. 9.- Pulsar J1312-5402 was detected outside the HPBW (marked by bigger circle) and $49^{\prime}$ offset (marked by smaller circle) from the survey pointing centre with $17 \sigma$ pulsed detection significance. The interferometric flux density is $22 \mathrm{mJy}$. The right plot shows a small portion of the image containing the pulsar.

Table 5: Parameters of the pulsars discovered in GHRSS survey

\begin{tabular}{|l|c|c|c|c|}
\hline Pulsar name & $\begin{array}{c}\text { Period } \\
(\mathrm{ms})\end{array}$ & $\begin{array}{c}\text { Dispersion measure } \\
\left(\mathrm{pc} \mathrm{cm}^{-3}\right)\end{array}$ & $\begin{array}{c}\text { Detection significance } \\
(\sigma)\end{array}$ & $\begin{array}{c}\text { Flux density } \\
(\mathrm{mJy})\end{array}$ \\
\hline PSR J0418-4154 & 757.11 & 24.5 & 50 & 10.3 \\
PSR J0514-4407 & 302.2 & 15.4 & 42 & 9.7 \\
PSR J0702-4956 & 666.66 & 98.7 & 30 & 15.7 \\
PSR J0919-42 & 812.6 & 57.8 & 19 & 6.4 \\
PSR J1255-46 & 52.0 & 42.9 & 12 & 0.8 \\
PSR J1456-48 & 536.81 & 133.0 & 15 & 1.2 \\
PSR J1559-44 & 1169.89 & 122.0 & 8 & 1.7 \\
PSR J1708-52 & 449.62 & 102.6 & 9 & 4.4 \\
PSR J1947-43 & 180.94 & 29.9 & 17 & 1.6 \\
PSR J2144-5237 & 5.04 & 19.0 & 9 & 4.7 \\
\hline
\end{tabular}

$\dagger^{\dagger}$ : Flux density is without primary beam correction. 


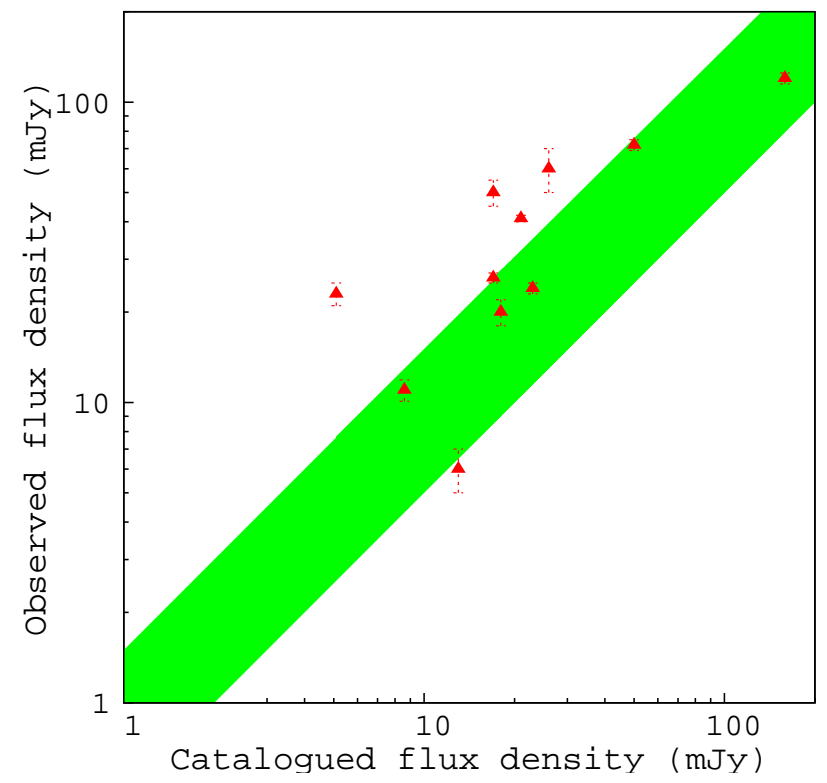

Fig. 10.- Comparison of catalogued mean flux density at $322 \mathrm{MHz}$ (extrapolated either by using the spectral index when available in the ATNF catalogue, or extracted considering 400 $\mathrm{MHz}$ mean flux density reported in the ATNF catalogue and a spectral index of -1.7) and observed interferometric flux density at $322 \mathrm{MHz}$ for 12 re-detected pulsars. The shaded part (green) represents a region with the observed flux density within $\pm 50 \%$ of the catalogued flux densities. 

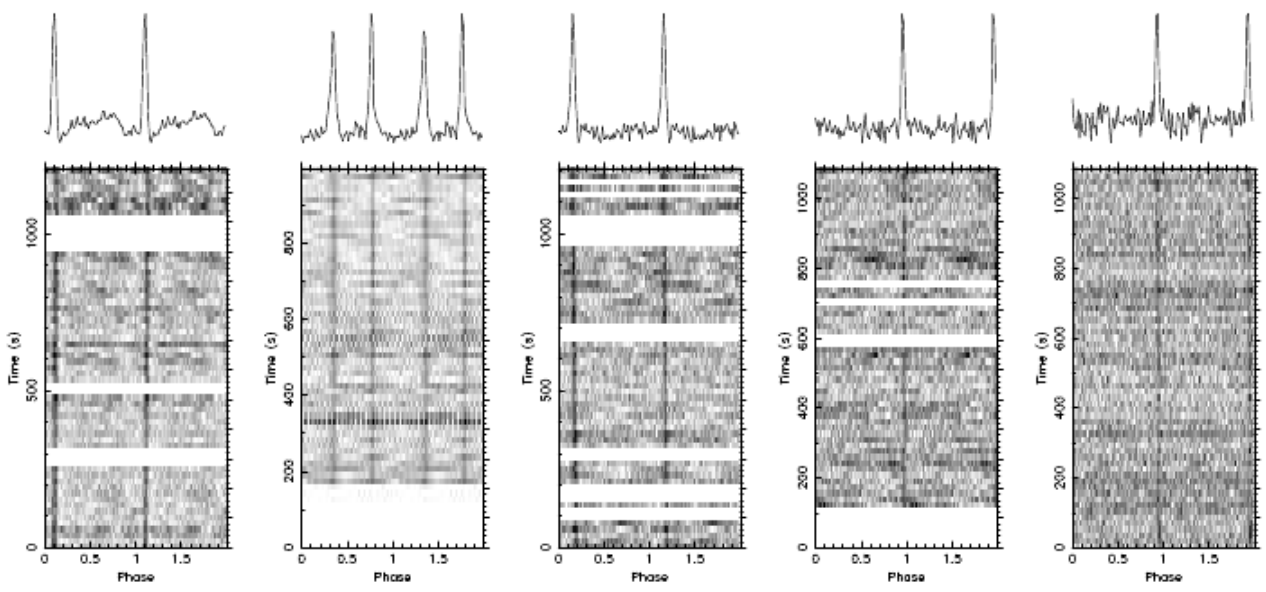

(a) J0418-4154

(b) J0514-4407

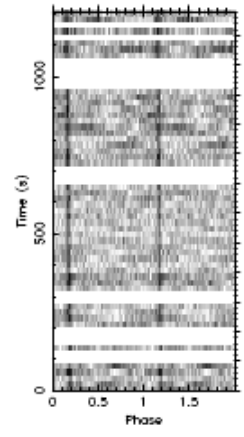

(d) J0919-42

(e) J1255-46
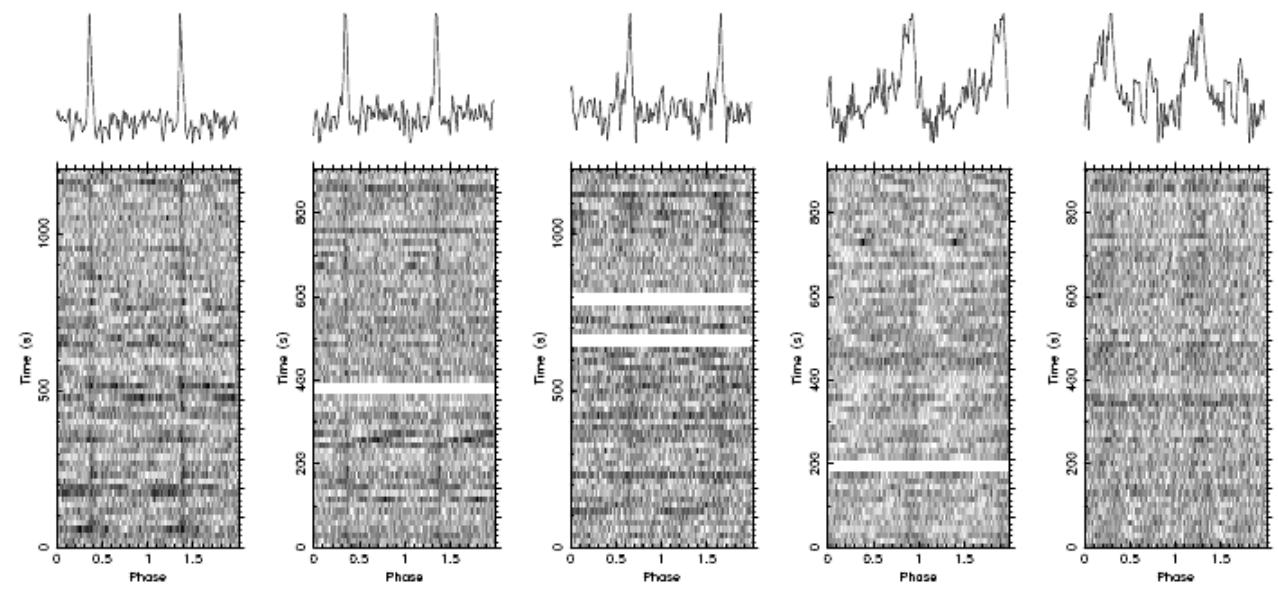

(f) J1456-48

(g) J1559-44

(h) J1708-52

(i) J1947-43

(j) J2144-5237

Fig. 11.- Discovery plots of the ten pulsars discovered in the GHRSS survey. The observations were performed in the incoherent array mode. The folded profiles are plotted (twice) in the top panel of each plot. Signal strength as a function of rotational phase is plotted in the bottom panel. Severe RFI occasions are masked, however as can be seen in some cases significant RFI remains. 
Table 6: List of 23 re-detected pulsars in the GHRSS survey. Offsets from the GHRSS beam centre, primary beam corrected pulsed SNR and interferometric flux densities (for 12 pulsars, the others either did not had overlapping SUMSS/NED observations, or could not be detected in the image due increased RMS near the pulsar position and/or significant flagging during imaging) are tabulated.

\begin{tabular}{|c|c|c|c|}
\hline Pulsar name & offset $\left(^{\prime}\right)$ & Pulsed-SNR & Interferometric Flux density (mJy) \\
\hline J0255-5304 & 25 & 31 & - \\
J0745-5353 & 72 & 272 & - \\
J0809-4753 & 69 & 176 & - \\
J0842-4851 & 53 & 60 & - \\
J0840-5332 & 53 & 30 & - \\
J0843-5022 & 0.0 & 11 & $12 \pm 1$ \\
J0905-5127 & 9 & 28 & - \\
J0907-5157 & 51 & 130 & $72 \pm 3$ \\
J0924-5302 & 26 & 77 & $26 \pm 1$ \\
J0934-5249 & 54 & 46 & $60 \pm 10$ \\
J0955-5304 & 62 & 132 & - \\
J1003-4747 & 2.5 & 82 & $11.0 \pm 0.9$ \\
J1036-4926 & 43 & 52 & $6.0 \pm 2$ \\
J1045-4509 & 33 & 50 & $24 \pm 1$ \\
J1143-5158 & 40 & 64 & - \\
J1240-4124 & 36 & 93 & $23 \pm 2$ \\
J1312-5402 & 58 & 43 & $41 \pm 1$ \\
J1320-5359 & 79 & 428 & $20 \pm 2$ \\
J1355-5153 & 47 & 114 & $50 \pm 5$ \\
J1544-5308 & 15 & 36 & - \\
J1559-4438 & 3.7 & 59 & $120 \pm 5$ \\
J1902-5105 & 75 & 159 & - \\
J2241-5236 & 13 & 71 & - \\
\hline
\end{tabular}



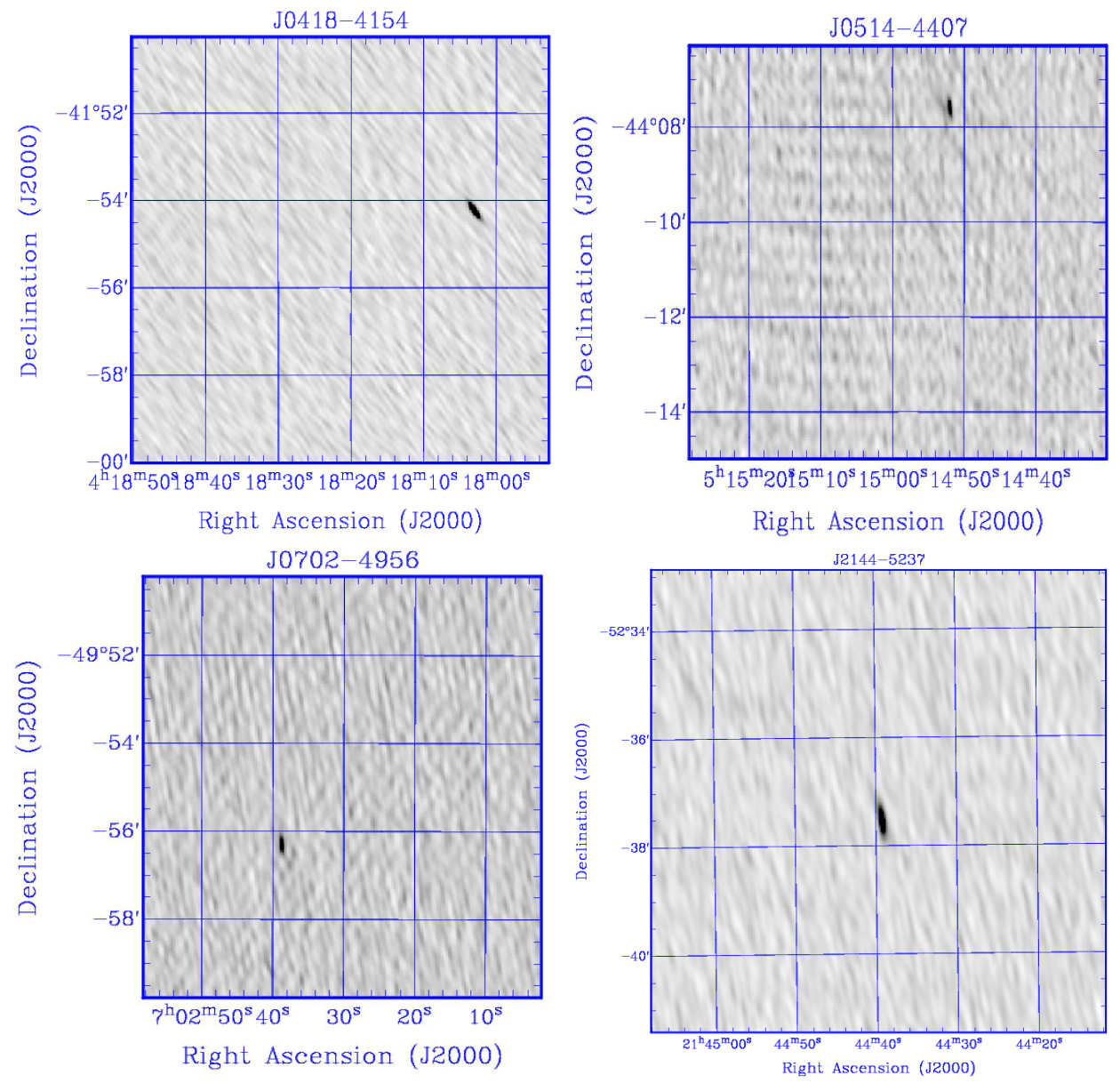

Fig. 12.- Localisation images for 4 pulsars discovered in the GHRSS survey: J0418-4154, J0514-4407, J0702-4956 and J2144-5237. The offsets of the pulsar from the pointing centre is given in Table 7. Since J0514-4407 and J2144-5237 are at large offsets from the pointing centre, we show a smaller field closer to the pulsar. 
Table 7: Parameters obtained from the gated imaging

\begin{tabular}{|c|c|c|c|c|c|c|}
\hline PSR & $\begin{array}{c}\text { Gated J2000 } \\
\text { position } \\
\left({ }^{\prime \prime}\right)\end{array}$ & $\begin{array}{l}\text { Offset from } \\
\text { pointing } \\
\text { centre }\end{array}$ & $\begin{array}{l}\text { Number } \\
\text { of gates }\end{array}$ & $\begin{array}{c}\text { Observing } \\
\text { duration } \\
\text { (s) }\end{array}$ & $\begin{array}{c}\text { Gated } \\
\text { flux density } \\
(\mathrm{mJy})\end{array}$ & $\begin{array}{c}\text { Gated } \\
\text { SNR }\end{array}$ \\
\hline J0418-4154 & $\begin{array}{l}04^{\mathrm{h}} 18^{\mathrm{m}} 02^{\mathrm{s}} .88\left(1^{\prime \prime} .4\right) \\
-41^{\circ} 54^{\prime} 11^{\prime \prime} .89\left(7^{\prime \prime} .8\right)\end{array}$ & $7.4^{\prime}$ & 24 & 900 & 88 & 9 \\
\hline J0514-4407 & $\begin{array}{l}05^{\mathrm{h}} 14^{\mathrm{m}} 51^{\mathrm{s}} .84\left(1^{\prime \prime} .04\right) \\
-44^{\circ} 07^{\prime} 06^{\prime \prime} .51\left(8^{\prime \prime} .4\right)\end{array}$ & $32^{\prime}$ & 21 & 900 & 20 & 5 \\
\hline J0702-4956 & $\begin{array}{l}07^{\mathrm{h}} 02^{\mathrm{m}} 38^{\mathrm{s}} .54\left(1^{\prime \prime} .2\right) \\
-49^{\circ} 56^{\prime} 56^{\prime \prime} .27\left(7^{\prime \prime} .8\right)\end{array}$ & $6.2^{\prime}$ & 16 & 900 & 29 & 6 \\
\hline $\mathrm{J} 2144-5237$ & $\begin{array}{l}21^{\mathrm{h}} 44^{\mathrm{m}} 39^{\mathrm{s}} .2\left(0^{\prime \prime} \cdot 7\right) \\
-52^{\circ} 37^{\prime} 32^{\prime \prime} .17\left(3^{\prime \prime} .8\right)\end{array}$ & $28^{\prime}$ & 10 & 3600 & 6 & 12 \\
\hline
\end{tabular}

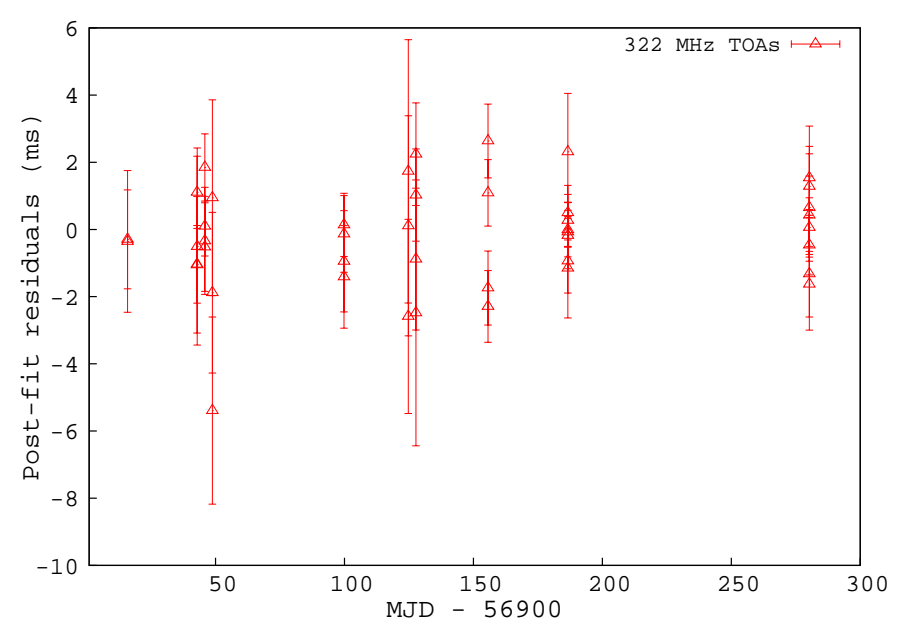

(a)

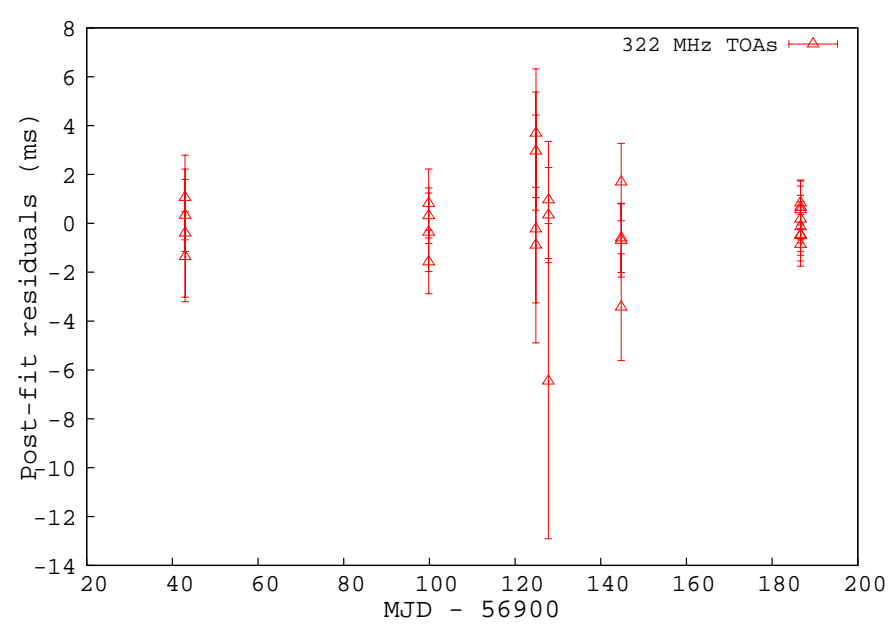

(b)

Fig. 13. - Post-fit timing residuals for J0418-4154 (left panel) and J0702-4956 (right panel). Multiple TOAs are derived for each observing epoch. Post-fit residual is $1.1 \mathrm{~ms}$ for J0418-4154 and $1.0 \mathrm{~ms}$ for J0702-4956. 


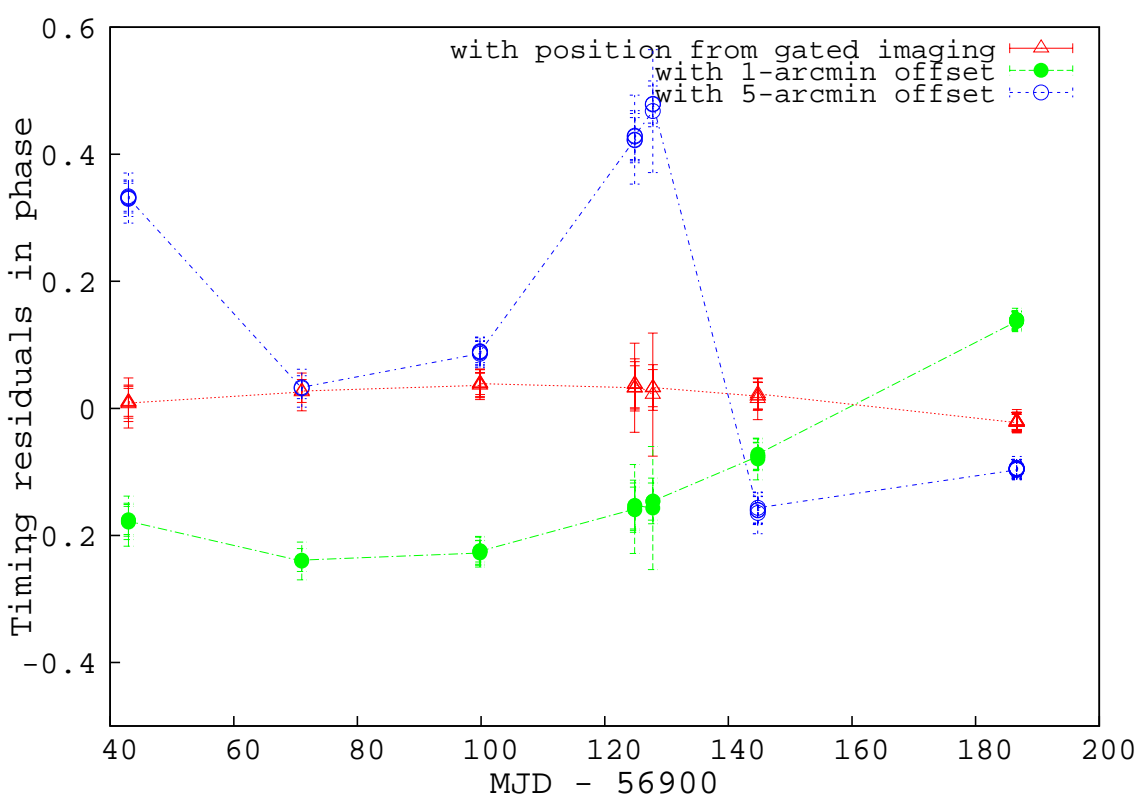

Fig. 14. - The post-fit timing residual for PSR J0702-4956 fitted with three timing models having different astrometric positions, the gated interferometric position (solid red line), $1^{\prime}$ offset (green dashed line) and $5^{\prime}$ offset (blue dotted line) from the gated interferometric position. The errors on TOAs are magnified by $10 \times$ for better visualisation. A \pm 0.5 phase wrap is noticed for the timing residual with $5^{\prime}$ position offset. 
Table 8: Timing parameters of PSR J0418-4154 and J0702-4956

\begin{tabular}{|c|c|c|}
\hline Parameters & J0418-4154 & J0702-4956 \\
\hline \multicolumn{3}{|c|}{ Gated imaging position } \\
\hline Right ascension(J2000) & $04^{\mathrm{h}} 18^{\mathrm{m}} 02^{\mathrm{s}} .88 \pm 1^{\mathrm{s}} .4$ & $07^{\mathrm{h}} 02^{\mathrm{m}} 38^{\mathrm{s}} .54 \pm 1^{\mathrm{s}} .2$ \\
\hline Declination(J2000) & $-41^{\circ} 54^{\prime} 11^{\prime \prime} .89 \pm 7^{\prime \prime} .8$ & $-49^{\circ} 56^{\prime} 56^{\prime \prime} \cdot 27 \pm 7^{\prime \prime} .8$ \\
\hline \multicolumn{3}{|c|}{ Parameters from radio-timing $^{a}$} \\
\hline Right ascension(J2000) ............ & $04^{\mathrm{h}} 18^{\mathrm{m}} 04^{\mathrm{s}} \cdot 2(2)$ & $07^{\mathrm{h}} 02^{\mathrm{m}} 39^{\mathrm{s}} \cdot 3(2)$ \\
\hline Declination(J2000) & $-41^{\circ} 54^{\prime} 10^{\prime \prime} 8(6)$ & $-49^{\circ} 56^{\prime} 34^{\prime \prime} .2(6)$ \\
\hline Pulsar frequency $f(\mathrm{~Hz})$. & $1.3207965590(1)$ & $1.501515953(1)$ \\
\hline Pulsar frequency derivative $\dot{f}\left(\mathrm{~Hz} \mathrm{~s}^{-1}\right)$ & $-1.7(1) \times 10^{-15}$ & 0.00 \\
\hline Period epoch(MAD) ............. & 57055 & 57086 \\
\hline Dispersion measure $\mathrm{DM}\left(\mathrm{pc} \mathrm{cm}^{-3}\right) \ldots$ & $24.54(8)$ & $98.7(1)$ \\
\hline Span of timing data(MAD). & $56915.88-57180.25$ & $56942.95-57086.64$ \\
\hline Number of TOAs & 44 & 29 \\
\hline Post-fit residual rms(ms) & 1.1 & 1.00 \\
\hline Reduced chi-square... & 1.05 & 0.7 \\
\hline
\end{tabular}

${ }^{a}$ Errors in the last digit are in parentheses 